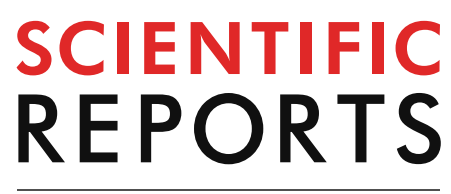

natureresearch

Check for updates

\title{
Enhancing microtubule
} stabilization rescues cognitive deficits and ameliorates pathological phenotype in an amyloidogenic Alzheimer's disease model

Juan Jose Fernandez-Valenzuela 1,2,5, Raquel Sanchez-Varo 1,2,5, Clara Muñoz-Castro 2,3,4 Vanessa De Castro ${ }^{1}$, Elisabeth Sanchez-Mejias ${ }^{1,2}$, Victoria Navarro ${ }^{2,3,4}$, Sebastian Jimenez ${ }^{2,3,4}$, Cristina Nuñez-Diaz ${ }^{1,2}$, Angela Gomez-Arboledas ${ }^{1,2}$, Ines Moreno-Gonzalez ${ }^{1,2}$, Marisa Vizuete ${ }^{2,3,4}$, Jose Carlos Davila ${ }^{1,2}$, Javier Vitorica ${ }^{2,3,4,6 \unrhd ~ \& ~ A n t o n i a ~ G u t i e r r e z ~}{ }^{1,2,6 \bowtie}$

In Alzheimer's disease (AD), and other tauopathies, microtubule destabilization compromises axonal and synaptic integrity contributing to neurodegeneration. These diseases are characterized by the intracellular accumulation of hyperphosphorylated tau leading to neurofibrillary pathology. AD brains also accumulate amyloid-beta $(A \beta)$ deposits. However, the effect of microtubule stabilizing agents on $A \beta$ pathology has not been assessed so far. Here we have evaluated the impact of the brain-penetrant microtubule-stabilizing agent Epothilone $\mathrm{D}$ (EpoD) in an amyloidogenic model of AD. Three-month-old APP/PS1 mice, before the pathology onset, were weekly injected with EpoD for 3 months. Treated mice showed significant decrease in the phospho-tau levels and, more interesting, in the intracellular and extracellular hippocampal $A \beta$ accumulation, including the soluble oligomeric forms. Moreover, a significant cognitive improvement and amelioration of the synaptic and neuritic pathology was found. Remarkably, EpoD exerted a neuroprotective effect on SOM-interneurons, a highly AD-vulnerable GABAergic subpopulation. Therefore, our results suggested that EpoD improved microtubule dynamics and axonal transport in an AD-like context, reducing tau and $A \beta$ levels and promoting neuronal and cognitive protection. These results underline the existence of a crosstalk between cytoskeleton pathology and the two major AD protein lesions. Therefore, microtubule stabilizers could be considered therapeutic agents to slow the progression of both tau and $A \beta$ pathology.

In Alzheimer's disease (AD), and other neurodegenerative tauopathies, tau protein is abnormally hyperphosphorylated and aggregated into intraneuronal neurofibrillary tangles (NFTs) ${ }^{1}$. AD is also characterized by the presence of extracellular amyloid plaques, composed predominately of amyloid- $\beta(A \beta)$ peptides, along with microglial response and neuroinflammation, axonal/synaptic damage and neuronal $\operatorname{loss}^{2-7}$.

${ }^{1}$ Dpto. Biología Celular, Genética y Fisiología, Instituto de Investigación Biomédica de Málaga-IBIMA, Facultad de Ciencias, Universidad de Málaga, Campus de Teatinos, 29071 Málaga, Spain. ${ }^{2}$ Centro de Investigación Biomédica en Red sobre Enfermedades Neurodegenerativas (CIBERNED), Madrid, Spain. ${ }^{3}$ Dpto. Bioquímica y Biología Molecular, Facultad de Farmacia, Universidad de Sevilla, C/Prof. Garcia Gonzalez 2, 41012 Sevilla, Spain. ${ }^{4}$ Instituto de Biomedicina de Sevilla (IBIS), Hospital Universitario Virgen del Rocio/CSIC, Universidad de Sevilla, Sevilla, Spain. ${ }^{5}$ These authors contributed equally: Juan Jose Fernandez-Valenzuela and Raquel Sanchez-Varo. ${ }^{6}$ These authors jointly supervised this work: Javier Vitorica and Antonia Gutierrez. ${ }^{\boxplus}$ email: vitorica@us.es; agutierrez@ uma.es 
a

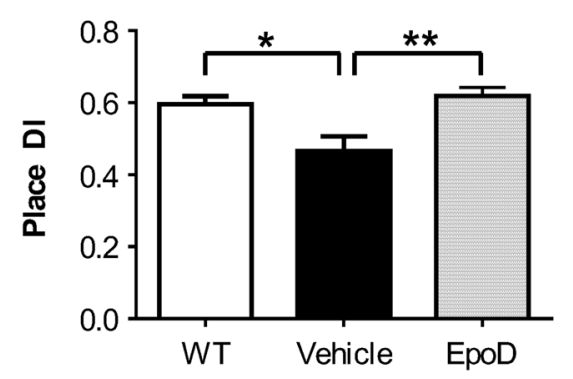

b

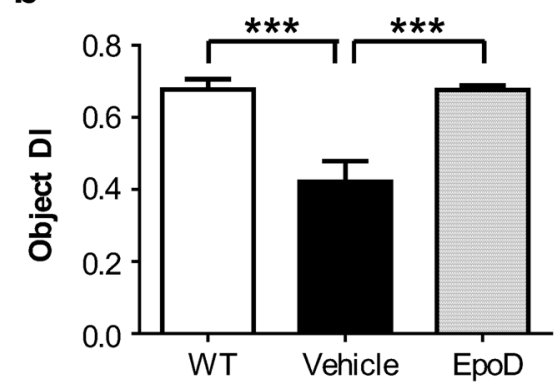

C

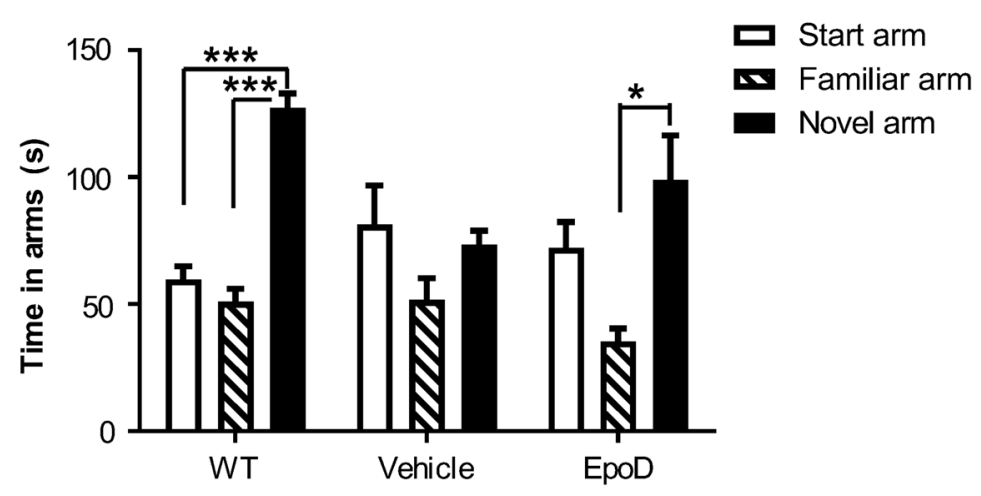

d

d1 Vehicle vs. WT ${ }^{*} p<0.05,{ }^{* *} p<0.01$

Vehicle vs. EpoD \#p<0.05, \#p $<0.01$

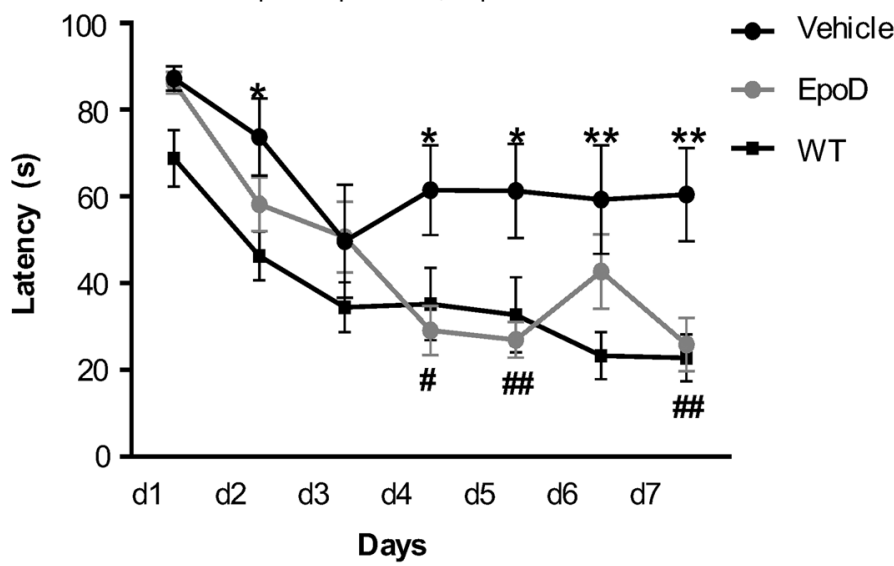

d2

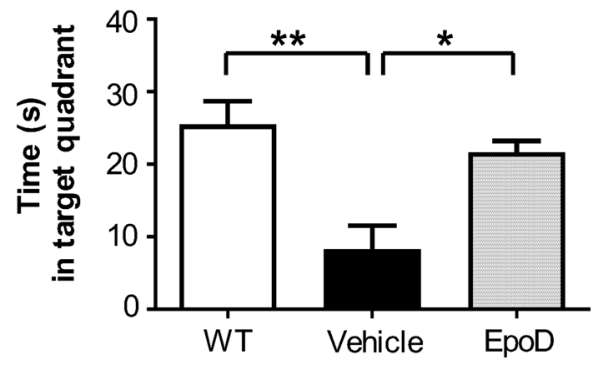


४Figure 1. EpoD treatment improved cognitive function in APP/PS1 mice. (a) At old-location test, one-way ANOVA $(\mathrm{F}(2,22)=7.842, \mathrm{p}=0.003)$ revealed significant differences between groups in place discrimination index (place DI). Post-hoc analysis showed that task performance of APP/PS1 ${ }^{\text {Veh }}$ animals (Tukey, ${ }^{*} \mathrm{p}<0.05$, $\left.{ }^{* *} \mathrm{p}<0.01\right)$ exhibited significant differences compared with WT or APP/PS1 ${ }^{\mathrm{EpoD}}$ mice. (b) Similarly, at novel object recognition task, one-way ANOVA $(F(2,22)=16.47, \mathrm{p}<0.0001)$ unveiled significant differences between groups in object DI. Post-hoc analysis showed a significant better performance in EpoD animals or WT mice, in comparison to vehicle (Tukey, ${ }^{* *} \mathrm{p}<0.001$ ). (c) Time spent in each arm during the retention phase of the Y-maze specific context test. One-way ANOVA of repeated measures (RM) was performed for each group. No arm preferences were found in APP/PS1 ${ }^{\text {Veh }}$ mice $(\mathrm{F}(2,10)=3.075, \mathrm{p}=0.13)$, in contrast to $\mathrm{EpoD}(\mathrm{F}(2,14)=5.15$, $\mathrm{p}=0.046)$ and WT $(\mathrm{F}(2,18)=41.933, \mathrm{p}=0.0001)$ groups. Post-hoc analysis determined that both APP/PS1 ${ }^{\mathrm{EpoD}}$ (Tukey, ${ }^{*} \mathrm{p}<0.05$ ) and WT (Tukey, ${ }^{* * *} \mathrm{p}<0.0001$ ), but not the vehicle group, spent more time in the novel than in the familiar arm (time in novel arm $F(2,21)=5.56, p=0.011)$. (d) Spatial learning and memory were evaluated in the Morris Water Maze using a 7-day hidden platform acquisition (4 trials/day). (d1) All groups decreased scape latency over the training. Two-way ANOVA revealed significant differences along learning days $(\mathrm{F}(6,126)=23.05, \mathrm{p}<0.001)$ and between groups $(\mathrm{F}(2,126)=5.81, \mathrm{p}<0.01)$. In addition, there was a significant interaction between the treatment and the latency $(\mathrm{F}(12,126)=2.138, \mathrm{p}=0.019)$. Post-hoc analysis showed poorer learning of vehicle animals as compared with either EpoD or WT groups (Tukey, EpoD vs. vehicle ${ }^{\#} \mathrm{p}<0.05$, ${ }^{\# \#} \mathrm{p}<0.01$; WT vs. vehicle $\left.{ }^{*} \mathrm{p}<0.05,{ }^{* *} \mathrm{p}<0.01\right)$. No differences were found between APP $/ \mathrm{PS} 1^{\mathrm{EpoD}}$ and WT. $(\mathbf{d} 2)$ In the probe test, one-way ANOVA $(\mathrm{F}(2,20)=9.34, \mathrm{p}=0.014)$ showed that both APP/PS1 ${ }^{\mathrm{EpoD}}$ and WT exhibited similar preference for the target quadrant in contrast to vehicle transgenic mice. Post-hoc testing determined that APP/PS $1^{\text {Veh }}$ spent less time swimming in the target quadrant than EpoD and WT groups (Tukey, ${ }^{\star} \mathrm{p}<0.05,{ }^{\star *} \mathrm{p}<0.01$, respectively). $s$ seconds. All data shown correspond to mean $\pm \mathrm{SEM}$.

The structural integrity and function of axonal microtubules (MT) depends on the microtubule-associated protein tau ${ }^{8,9}$. Since neurons are extremely polarized cells from both a morphological and functional point of view, they largely depend on transport along their axons to be completely healthy and well-functioning ${ }^{10,11}$, which in turn relies on the proper binding of tau to tubulins ${ }^{12}$. Significantly, a number of axonal-flow dependent mechanisms have been reported to be disturbed in neurons from $\mathrm{AD}$ patients and models ${ }^{13-16}$, which exhibit intra/extracellular $A \beta$ accumulation, intraneuronal aberrant aggregates of hyperphosphorylated (phospho)-tau, dystrophic neurites and interferences in anterograde/retrograde transport. Therefore, it is not surprising that both gain-of-toxicity and loss-of-function of tau, together with the consequent microtubular destabilization, have arisen as novel putative pharmacological targets ${ }^{17-20}$. Thus, MT-targeting agents are attractive therapeutic candidates for neurodegenerative diseases ${ }^{20,21}$. In fact, different MT-stabilizing compounds like taxanes or epothilones, used in anticancer chemotherapy, have been recently assessed in some preclinical studies using transgenic models of tauopathy ${ }^{22,23}$. Specifically, Epothilone D (EpoD) is a brain-penetrant drug that has arisen as a good candidate to test the potential of microtubule stabilizers as a treatment for neurodegenerative diseases. In fact, several drugs that affect microtubule stability are currently under investigation as potential treatments for $\mathrm{AD}^{18,20}$. However, as far as we are concerned, to date the assessment of MT-stabilizing compounds has not been evaluated in amyloidogenic AD models and the impact of this therapeutic approach on amyloid-associated pathology remains to be dilucidated.

In this work we have evaluated the effect of intraperitoneal administration of EpoD in a double-transgenic mouse model, APP/PS1, starting before the onset of the pathology (from 3 to 6 months of age). The hippocampus of these mice exhibits significant amyloid extracellular deposits which are decorated with axonal/synaptic dystrophic neurites ${ }^{24-31}$. Moreover, this model develops an important GABAergic neuronal loss at early ages in hippocampus, entorhinal and perirhinal cortices ${ }^{30-35}$. Though these mice do not reproduce the formation of NFTs, they develop pathological posttranslational modifications of tau leading to AT8-positive aggregates within dystrophic neurites showing a connection between $A \beta$ and tau pathology. Our data indicated that microtubule stabilization induced by EpoD treatment promoted memory improvement, synaptic/neuritic recovery and neuronal protection. Furthermore, EpoD treatment reduced not only tau pathology, but more interestingly, the intra- and extracellular A $\beta$ accumulation. Thus, MT-stabilizing compounds may be used for targeting both $\mathrm{A} \beta$ and tau pathology in $\mathrm{AD}$ treatment.

\section{Results}

EpoD rescues memory deficits in APP/PS1 mice. We first tested whether EpoD treatment prevents/ ameliorates cognitive deficiencies in our amyloidogenic model. APP/PS1 ${ }^{\mathrm{EpoD}}$ and APP/PS1 ${ }^{\text {Veh }}$ mice were evaluated the last 2 weeks of treatment. Non-treated age-matched WT mice were also included for comparison purposes. Open-field test (OFT) was performed to determine baseline activity as well as to evaluate whether EpoD treatment or vehicle injections provokes alterations on the locomotor and exploratory behavior. No significant differences between the three groups were observed for either, the distance roamed, speed or the number of rearings (Supplemental Fig. S1). Therefore, APP/PS1 ${ }^{\mathrm{EpoD}}$ or APP/PS1 ${ }^{\mathrm{Veh}}$ mice did not display locomotive or exploratory behavior alterations when compared to age-matched WT animals. We next evaluated the effects of EpoD on episodic-like memory using the old-location task (OLT, spatial memory) and the novel object recognition task (NORT, recognition memory), utilizing the natural tendency of the animals to preferentially explore novel objects or placed in new locations (spontaneous exploratory behavior). OLT data (Fig. 1a) revealed that EpoD treatment protected from the cognitive deficits observed in vehicle-injected APP/PS1 group ( $\mathrm{p}<0.01)$. In fact, APP/PS1 ${ }^{\mathrm{EPoD}}$ mice performed indistinguishable from WT mice. Similarly, NORT data (Fig. 1b) indicated that APP/PS1 $1^{\text {Veh }}$ mice performed worse than APP/PS1 ${ }^{\mathrm{EpoD}}$ group $(\mathrm{p}<0.001)$, which in turn behaved similarly to WT mice, demonstrating a protective action of this compound on this type of memory. 
a

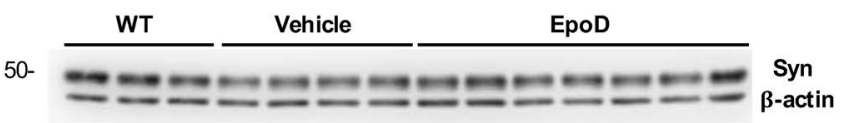

C

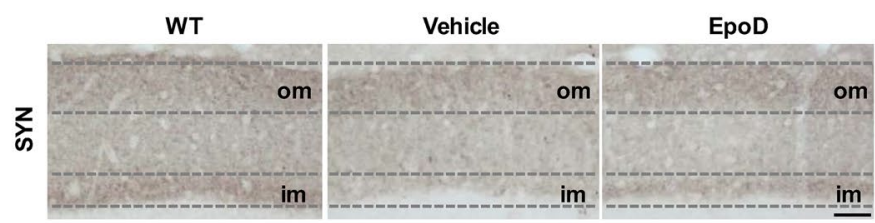

e

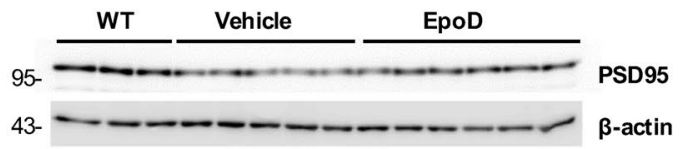

g

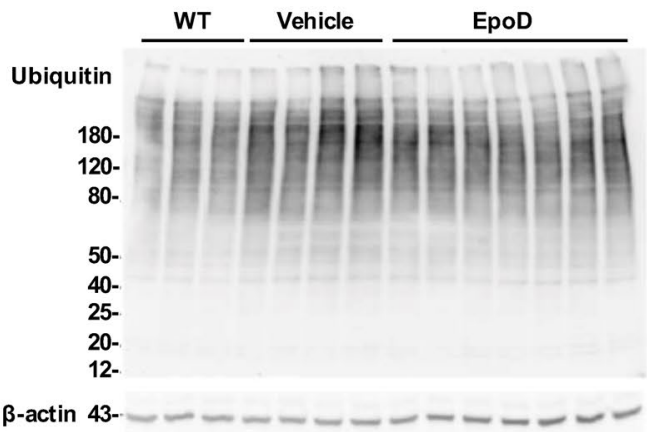

b

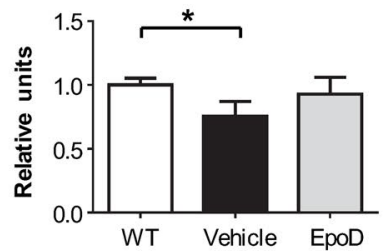

d
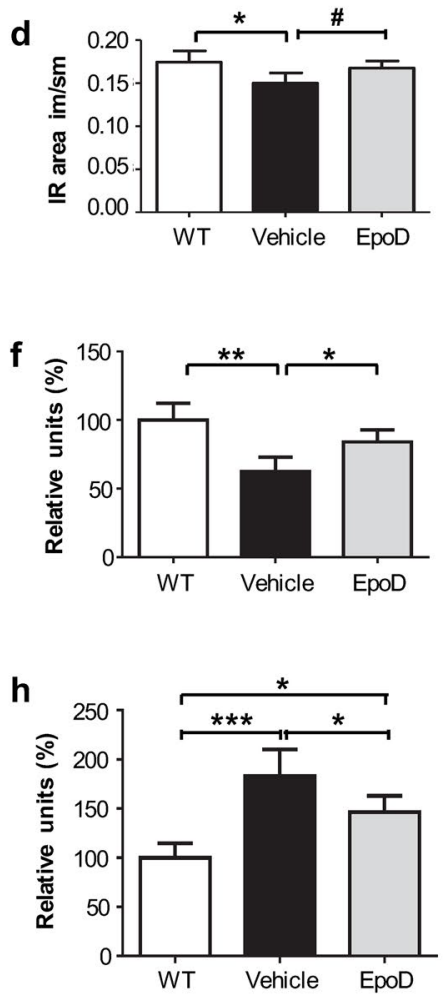
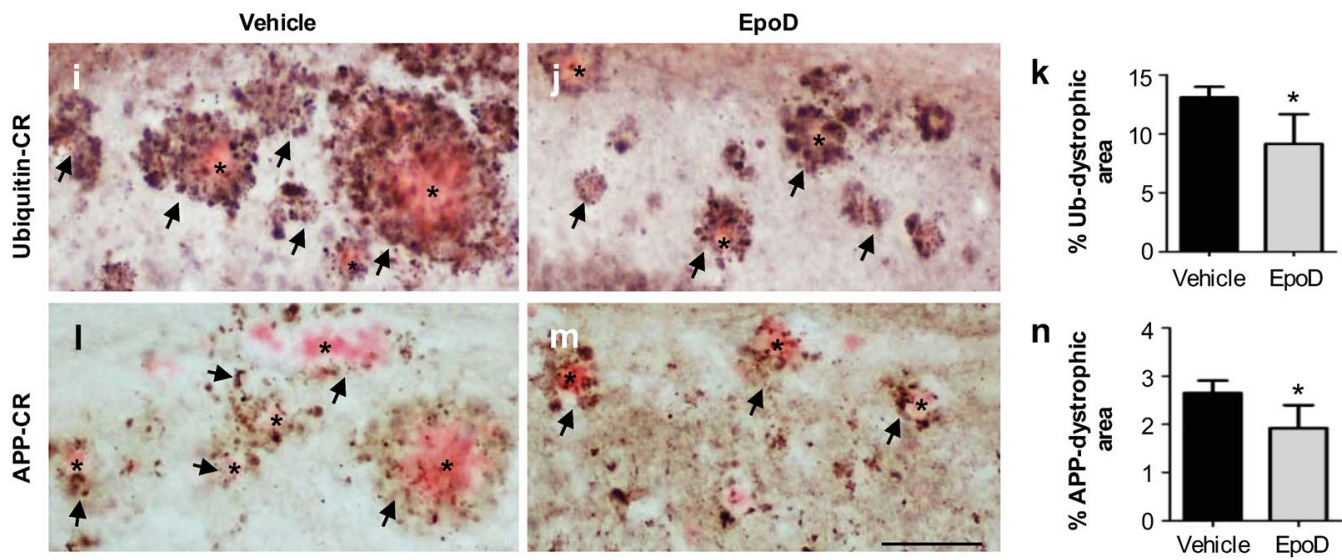
\Figure 2. EpoD treatment reduced both synaptic and plaque-associated neuritic pathology. (a,b) Representative western blot and quantitative analysis of synaptophysin (Syn) in total hippocampal proteins from WT $(n=3)$, vehicle $(n=4)$ and EpoD $(n=7)$ mice. For quantification, data were referred to WT group. $\beta$-Actin was used as the loading control. Syn protein levels were decreased in vehicle group and recovered in EpoD mice (ANOVA $F(2,11)=4.386, p=0.039$, Tukey, $\left.{ }^{*} \mathrm{p}<0.05\right)$. (c) Syn-immunostaining shows a laminar pattern in the molecular layer of the hippocampal dentate gyrus. (d) The area occupied by the Syn-positive inner band of the molecular layer (im) in APP/PS1 ${ }^{\mathrm{EpoD}}$ animals was similar to that in WT mice, and significant bigger than in APP/PS1 $1^{\text {Veh }}$ mice (ANOVA F $(2,12)=5.644, \mathrm{p}=0.019$, Tukey, $\left.{ }^{*} \mathrm{p}<0.05\right)$. $(\mathbf{e}, \mathbf{f})$ Representative western blot and quantitative analysis of the postsynaptic marker PSD95 in total hippocampal proteins from WT, vehicle and EpoD mice. For quantification, data were referred to WT group. $\beta$-Actin was used as the loading control. PSD95 protein levels were significantly recovered in EpoD group (ANOVA $(F(2,10)=12.44, p=0.0019$, Tukey, ${ }^{*} \mathrm{p}<0.05$ ). (g,h) Quantitative western blot for ubiquitin (ANOVA $\mathrm{F}(2,11)=14.97, \mathrm{p}<0.001$ ) showed a significant increase in vehicle mice (Tukey, ${ }^{* *} p<0.001$ ) and a significant reduction (Tukey, ${ }^{*} \mathrm{p}<0.05$ ) in the hippocampus of treated mice as compared with WT. It is noteworthy that EpoD reverted partially (as compared with WT, Tukey, $\left.{ }^{\star} \mathrm{p}<0.05\right)$ the ubiquitinated protein accumulation. Expression of the different markers were always referred to WT. Hippocampal sections were immunolabeled for ubiquitin $(\mathbf{i}, \mathbf{j})$ or APP $(\mathbf{l}, \mathbf{m})$, and counterstained with Congo red (CR), to evidence protein build-up within dystrophic neurites around amyloid plaques (asterisk, see details in insets). Quantitative image analysis demonstrated a significant decrease of (k) ubiquitin- ( $t$ test, $\left.{ }^{\star} \mathrm{p}<0.05\right)$ and $(\mathbf{n})$ APP-positive area $\left(t\right.$ test, $\left.{ }^{*} \mathrm{p}<0.05\right)$ in CA1 subfield in APP/PS1 ${ }^{\mathrm{EpoD}}$ in comparison to controls. Uncropped blots are shown in Supplementary Fig. S6. im inner molecular layer, om outer molecular layer, $s \mathrm{~s} m$ stratum lacunosum-moleculare, so stratum oriens, $s p$ stratum pyramidale, $s r$ stratum radiatum. Scale bars: (c) $50 \mu \mathrm{m} ;(\mathbf{i}, \mathbf{j})$ and $(\mathbf{l}, \mathbf{m}) 100 \mu \mathrm{m}$.

Long-term memory was analyzed using a variant of the Y-maze with patterned walls (Fig. 1c) to evaluate whether mice are able to distinguish the maze arms according to the time spent in each arm during the retention phase. APP/PS1 $1^{\mathrm{EpoD}}$ mice exhibited a significant preference for the novel arm compared to the familiar one $(\mathrm{p}<0.05)$, as the WT mice did $(\mathrm{p}<0.0001)$, in contrast to vehicle-injected mice which did not discriminate between novel and familiar arm. No significant differences in distance or speed were observed among groups (Supplemental Fig. S1).

The effect of EpoD treatment on learning and memory was also evaluated by Morris Water Maze (MWM; Fig. 1d). Navigational learning and memory skills were assessed using a 7-day hidden platform acquisition ( 4 trials/day). No visual defects were detected with visible platform task among groups (not shown). During acquisition, all groups learned to locate the hidden platform by spatial navigation, as demonstrated by decreases in latency (Fig. 1d1). Importantly, APP/PS1 ${ }^{\mathrm{EpoD}}$ mice needed less time to find the scape platform than the APP/ PS1 ${ }^{\text {Veh }}$ group $(\mathrm{p}<0.01)$, performing very similar to WT mice. Therefore, these results indicated that EpoD treatment was involved in the preservation of APP/PS1 allocentric learning. Afterwards, during the probe trial (retention phase) (Fig. 1d2), APP/PS1 ${ }^{\text {Veh }}$ mice displayed memory deficits, spending significant less time in the target zone $(\mathrm{p}<0.05)$. However, no differences were observed between APP/PS $1^{\mathrm{EpoD}}$ and WT mice, suggesting that EpoD treatment was also able to keep the short-term spatial memory ability in APP/PS1 mice.

Taken together, all these data indicated that EpoD treatment prevented the onset of hippocampal-related cognitive impairment in APP/PS1 mice.

EpoD improves microtubular stability in the hippocampus of APP/PS1. Next, to evaluate the microtubule-stabilizing effect of EpoD we analyzed the expression of acetylated tubulin (AcTub), a well-known biomarker of stable microtubules ${ }^{36}$. Tubulin acetylation takes place after polymerization into microtubules, and the most stable pools are known to be enriched in $\mathrm{AcTub}^{37,38}$. Immunofluorescence analysis of this marker by confocal microscopy evidenced a moderate/intense neuronal cell bodies, apical dendrites and axonal tracts in the hippocampus of WT mice (CA1 region and alveus are depicted in Supplemental Fig. S2). APP/PS1 ${ }^{\text {Veh }}$ mice exhibited lower AcTub immunoreactivity than WT mice, indicating a higher microtubule instability. Interestingly, a stronger immunostaining for AcTub was observed in APP/PS1 ${ }^{\mathrm{EPOD}}$ mice in comparison to the vehicle group, indicating a better preservation of microtubule integrity in the hippocampal neurons of the EpoD group.

EpoD protects against $A \beta$-induced synaptic/axonal pathology. As we have previously reported, the hippocampus of the APP/PS1 model develops prominent axonal/presynaptic pathology (plaque-associated dystrophic neurites formation) since early ages ${ }^{27-32,34}$. The functional integrity of axons/synapses depends on the axonal transport and, consequently, on microtubules. Thus, aiming to investigate whether the protection against the cognitive decline observed in APP/PS1 ${ }^{\mathrm{EPoD}}$ mice was due to a synaptic/axonal preservation, we analyzed the expression of synaptophysin (Syn) and PSD95, classic presynaptic and postsynaptic markers respectively. Western blot analysis (Fig. 2a,b) showed a significant decrease of Syn levels in APP/PS1 ${ }^{\text {Veh }}$ mice $(75.40 \pm 11.68 \%$ vs. WT mice, $\mathrm{p}<0.05)$, which was almost recovered by EpoD treatment $(92.86 \pm 13.19 \%$ vs. WT mice; 1.23 -fold increase respect to vehicle group). Next, we validated these data by immunostaining (Fig. 2c). Typically, in WT mice the outer and the inner layers of the dentate gyrus stratum moleculare showed a higher Syn immunoreactivity than the middle one (Fig. 2c), according to the spatial arrangements of entorhino-dentate projections ${ }^{39}$ together with local GABAergic afferents ${ }^{40}$. However, Syn-immunostaining (Fig. 2c,d) within the inner molecular layer in APP/PS $1^{\text {Veh }}$ dentate gyrus was reduced $(-14.28 \pm 6.9 \%$ vs. WT mice, $\mathrm{p}<0.05)$ whereas it was preserved in $\mathrm{APP} / \mathrm{PS} 1^{\mathrm{EpoD}}$ mice $(+10.28 \pm 5.53 \%$ vs. vehicle group, $\mathrm{p}<0.05)$. Accordingly, levels of the postsynaptic marker PSD95, measured by western blots (Fig. 2e,f), were significantly higher (1.34-fold) in the hippocampus of EpoD- 
WT
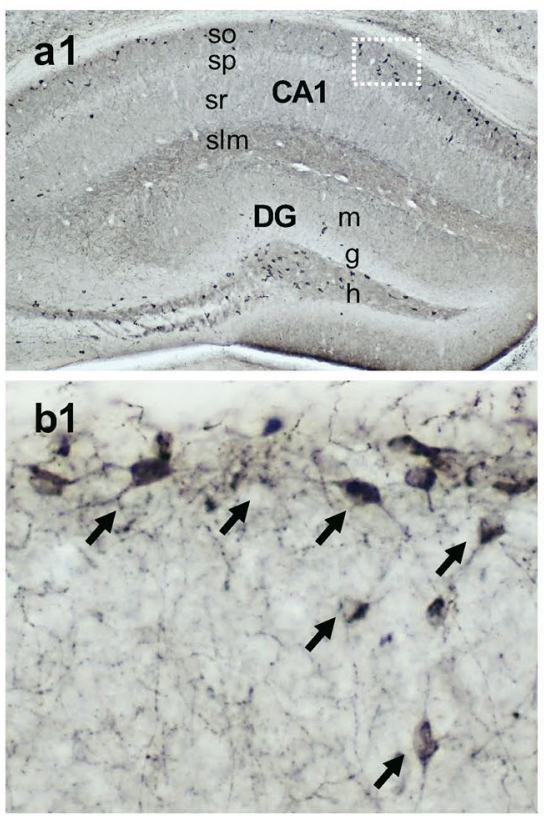

C

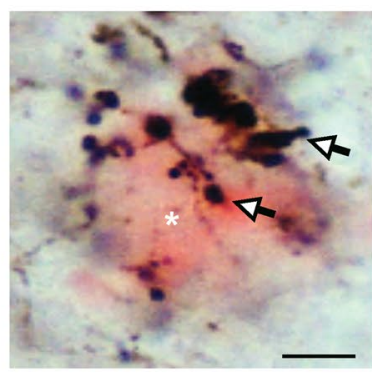

Vehicle

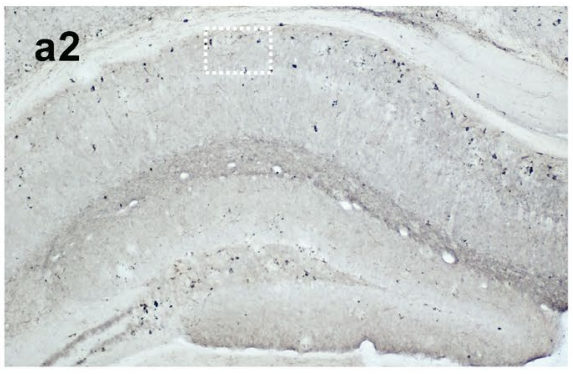

b2

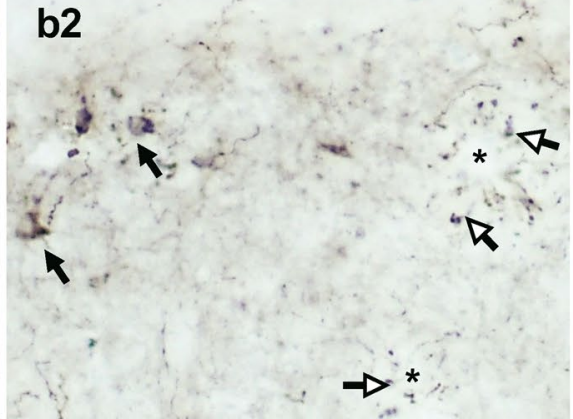

d

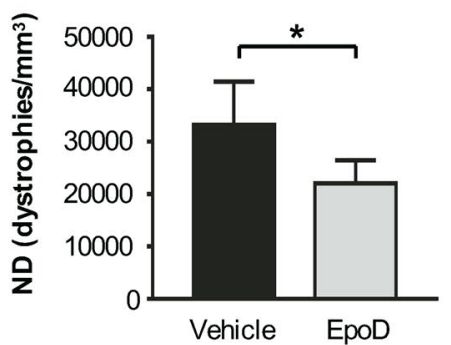

EpoD

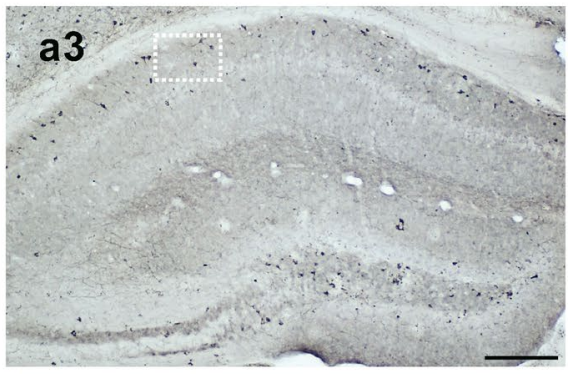

b3
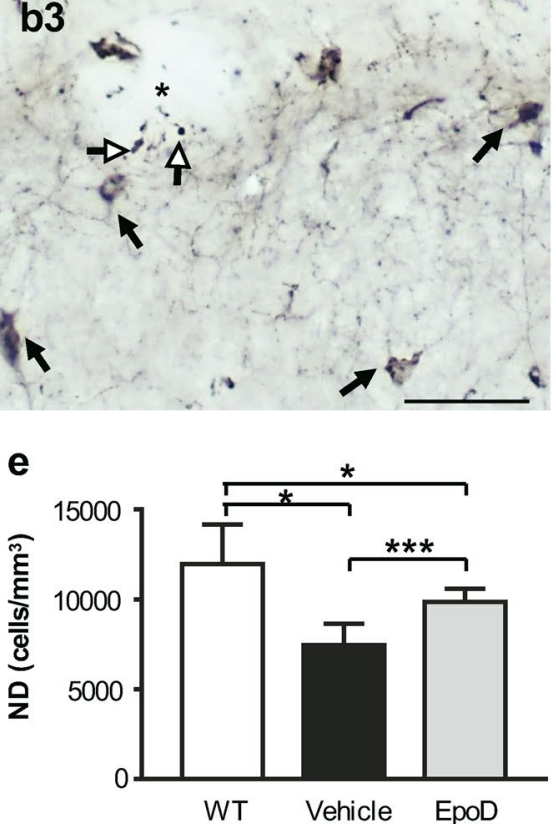

Figure 3. Epothilone D treatment ameliorated hippocampal SOM-interneuron degeneration. (a1-a3) Panoramic view of SOM-immunolabeled hippocampal sections of WT (a1), APP/PS1 ${ }^{\text {Veh }}$ (a2) and APP/PS1 ${ }^{\mathrm{EpoD}}$ (a3) mice. (b1-b3) Higher magnification of the stratum oriens from the CA1 subfield showing a reduction in the amount of SOM-positive somata (black arrows) and the corresponding neuropile in vehicle group (b2) in contrast to EpoD (b3) and WT (b1). (b2,b3) SOM-positive dystrophies in APP/PS1 mice were also evidenced (white arrows, neuritic dystrophies; asterisks, amyloid plaques). (c) As visualized by Congo red counterstaining, SOM-immunoreactive dystrophic neurites (white arrows) in APP/PS1 mice are associated with amyloid plaques (red). (d,e) Numerical densities (ND) of SOM-positive dystrophies and neurons in CA1 subfield were assessed by stereology. WT $(\mathrm{n}=4), \mathrm{APP} / \mathrm{PS} 1^{\mathrm{Veh}}(\mathrm{n}=4)$ and APP/PS1 ${ }^{\mathrm{EpoD}}(\mathrm{n}=7)$. (d) As expected, EpoD provoked a significant decrease in the amount of SOM-positive aberrant neurites in this region (EpoD vs. vehicle, $t$ test, ${ }^{\star} \mathrm{p}<0.05$ ). No SOM-positive dystrophies were observed in WT mice. (e) Quantitative analysis demonstrated a significant loss of SOM-interneurons in $\mathrm{APP} / \mathrm{PS} 1^{\mathrm{Veh}}$ and a partial neuroprotective effect in EpoD-treated group as compared with WT (ANOVA, $\mathrm{F}(2,12)=11.15 ; \mathrm{p}=0.0018$; Tukey, EpoD vs. vehicle ${ }^{* * *} \mathrm{p}<0.001$; vehicle vs. WT $\left.{ }^{*} \mathrm{p}<0.05\right)$. CA cornu Ammonis, $D G$ dentate gyrus, $h$ hilus, $g$ granular layer, $m$ stratum moleculare, slm stratum lacunosum-moleculare, so stratum oriens, $s p$ stratum pyramidale, $s r$ stratum radiatum. Scale bars: (a1-a3) $250 \mu \mathrm{m}$; (b1-b3) $50 \mu \mathrm{m}$; (c) $10 \mu \mathrm{m}$.

treated mice compared to vehicle group $(84.01 \pm 8.80 \%$ and $62.46 \pm 10.44 \%$, respectively; data compared to WT mice. EpoD vs. vehicle $\mathrm{p}<0.05$; WT vs. vehicle $\mathrm{p}<0.01)$.

This synapse protection could be a consequence of EpoD-mediated amelioration of the previously reported axonal pathology in this model ${ }^{28,29}$. Thus, we next assessed the accumulation of total ubiquitinated proteins and the ubiquitin-positive dystrophies in the hippocampus (Fig. 2g-k). Quantitative western blotting (Fig. 2g,h) revealed that the levels of ubiquitinated proteins were indeed increased in APP/PS1 ${ }^{\text {Veh }}$ mice $\left(182.83 \pm 27.38 \%, \mathrm{p}<0.001\right.$ as compared with WT animals) and consequently reduced in APP/PS1 $1^{\mathrm{EpoD}}$ hippocampus ( $146.42 \pm 16.54 \%, \mathrm{p}<0.05$ as compared with vehicle). These data were confirmed by ubiquitin immunolabeling combined with image analysis. As previously reported and showed in Fig. 2i,j, ubiquitin immunostaining accumulated in dystrophic neurites surrounding $\mathrm{A} \beta$ plaques. EpoD treatment produced a significant reduction $(-30.10 \pm 19.18 \%, \mathrm{p}<0.05)$ in the CA1 ubiquitin loading in APP/PS1 ${ }^{\mathrm{EpoD}}$ animals, compared to APP/PS1 ${ }^{\mathrm{Veh}}$ (Fig. 2k). Since dystrophies were also positive for APP-protein ${ }^{28}$, the EpoD-dependent reduction in dystrophic 
a

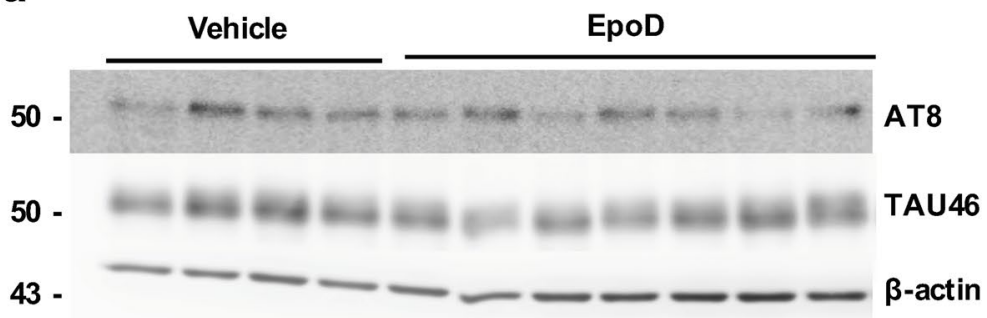

b

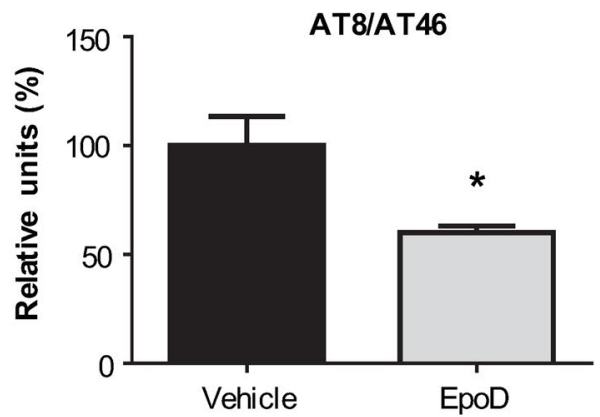

d
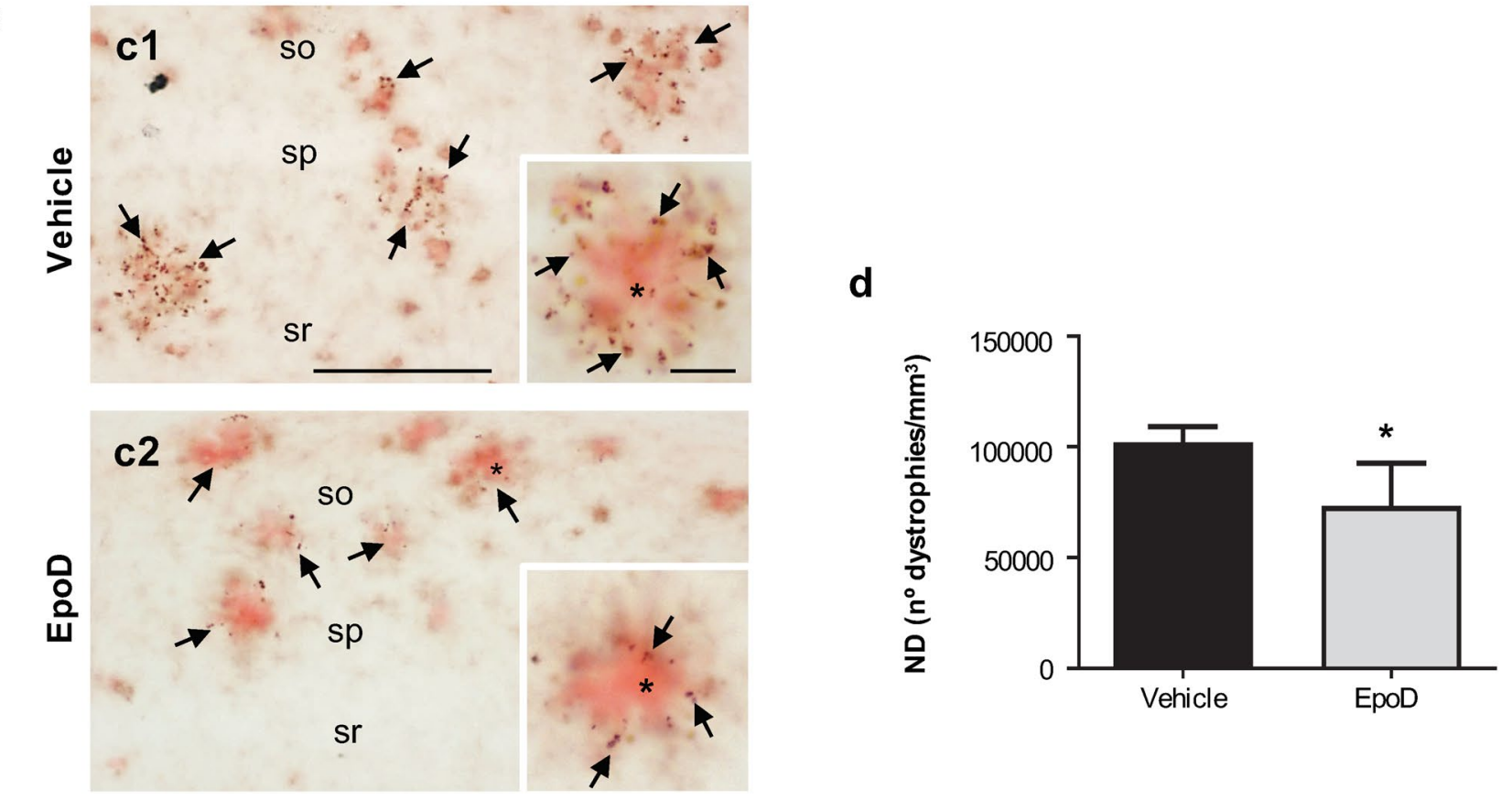

Figure 4. Tau pathology decreased in the hippocampus of EpoD-treated mice. (a,b) Representative western blot and quantitative analysis of the phospho-tau ratio (AT8/TAU46) from APP/PS1 ${ }^{\text {Veh }}(\mathrm{n}=4)$ and APP/PS1 $1^{\mathrm{EpoD}}$ $(n=7)$ mice. $\beta$-Actin was used as the loading control. For quantification, ratio was referred to vehicle group. The ratio of phospho-tau/total tau was significantly reduced in treated mice $\left(t\right.$ test, $\left.{ }^{*} \mathrm{p}<0.05\right)$. Uncropped blots are shown in Supplementary Fig. S7. (c) AT8-positive dystrophies (arrows) were located surrounding A $\beta$ plaques (Congo red, asterisk). Quantitative analysis (d) showed a significant decrease in the numerical density (ND) of AT8-positive dystrophic neurites $\left(t\right.$ test, $\left.{ }^{*} \mathrm{p}<0.05\right)$ in CA1 region from EpoD (c2) compared to vehicle mice $(\mathbf{c 1})$. so stratum oriens, $s p$ stratum pyramidale, $s r$ stratum radiatum. Scale bars: (c1) and (c2) $200 \mu \mathrm{m}$; insets $20 \mu \mathrm{m}$.

neurites was also corroborated using an anti-APP antibody. As shown (Fig. 2l-n), we observed a significant reduction $(-27.51 \pm 18.12 \%, \mathrm{p}<0.05)$ in the APP-loading surrounding $\mathrm{A} \beta$ plaques.

Therefore, EpoD treatment preserved the synaptic integrity, reduced the accumulation of ubiquitinated proteins and decreased the dystrophic pathology in this amyloidogenic model. All these neuroprotective effects should reflect an amelioration of the axonal transport defects by improving microtubular stability.

Microtubular stabilization reduces SOM interneuron loss. A significant loss of GABAergic SOM interneurons in memory-relevant brain regions is an early pathological event in this APP/PS1 model ${ }^{31,33-35}$. Therefore, we assessed the density of SOM-cells and SOM-positive dystrophic neurites in the CA1 subfield of hippocampus by stereological quantification (Fig. 3). As expected, APP/PS1 ${ }^{\text {Veh }}$ mice (Fig. 3a2,b2) showed abundant SOM-positive dystrophic neurites around amyloid plaques (Fig. 3c), and displayed a significant decrease $(-37.57 \pm 9.8 \%, \mathrm{p}<0.05)$ of SOM-positive neurons (Fig. 3e), compared to age-matched WT mice (Fig. 3a1,b1). Noteworthy, EpoD treatment (Fig. 3a3,b3) significantly reduced the amount of plaque-associated SOM-positive dystrophies $(-33.63 \pm 13.17 \%, \mathrm{p}<0.05$, compared to vehicle group; Fig. $3 \mathrm{~d})$. More relevant, the numerical density $\left(\right.$ cells $\left./ \mathrm{mm}^{3}\right)$ of SOM neurons was higher in APP/PS1 ${ }^{\text {EpoD }}(+20.07 \pm 9.73 \%, \mathrm{p}<0.001)$ than in vehicle group, though did not reach WT values (Fig. 3e). Thus, these data suggested that stabilization of microtubules by EpoD was able to protect, at least in part, this vulnerable hippocampal interneuron subpopulation not only from axonal damage but also from cell death in this APP/PS1 model. 
a

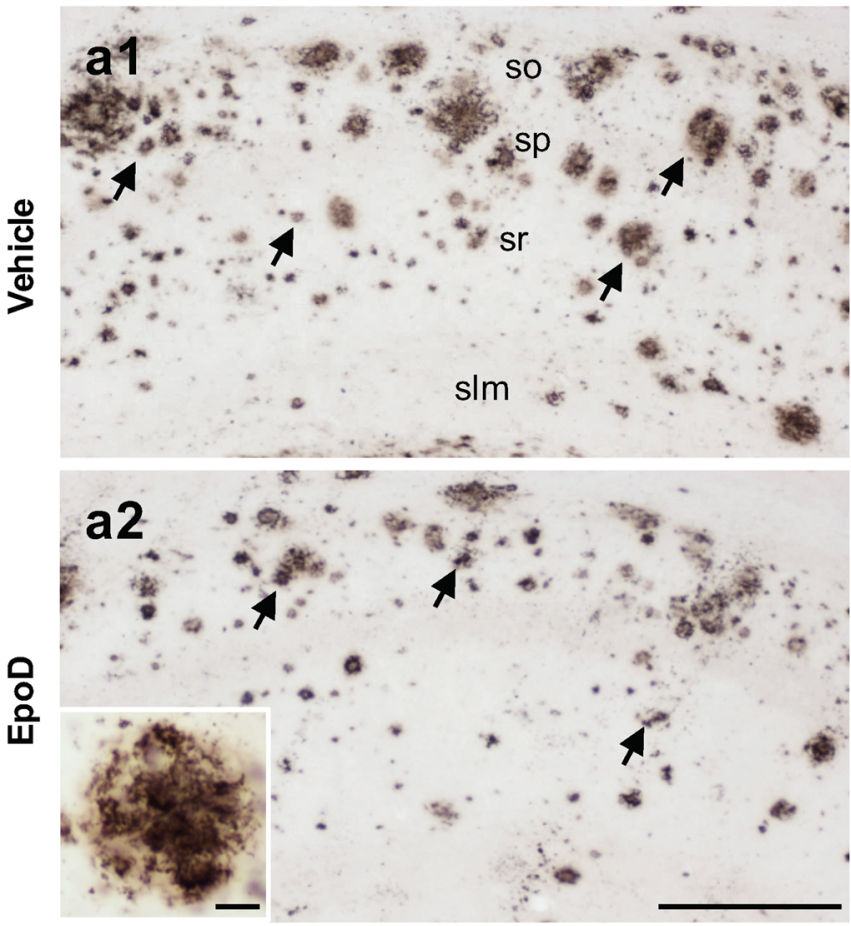

b

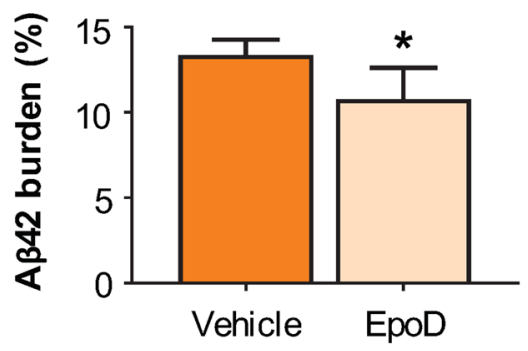

d

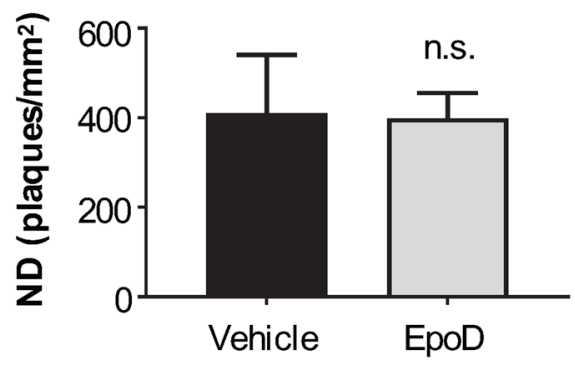

Thio-S
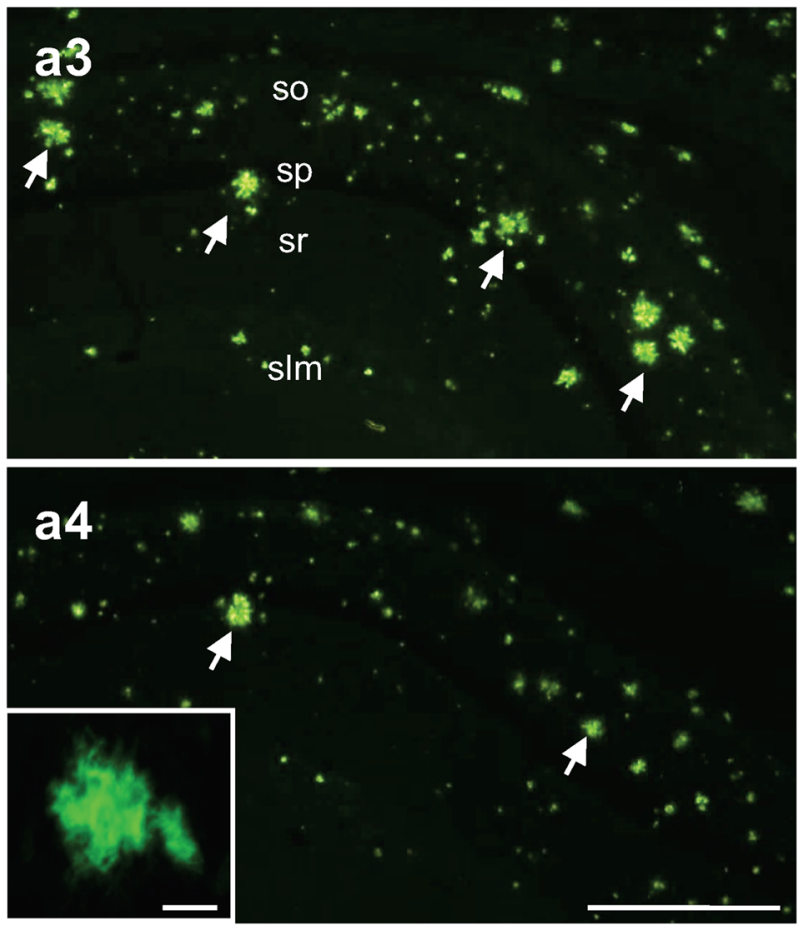

C

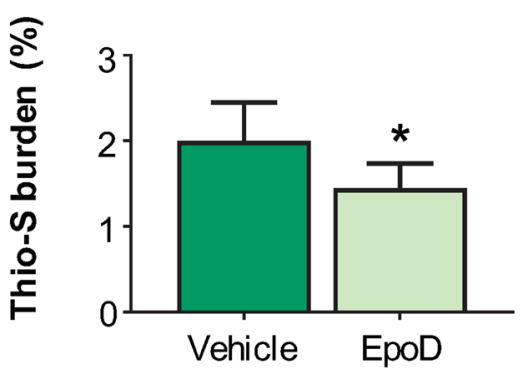

e

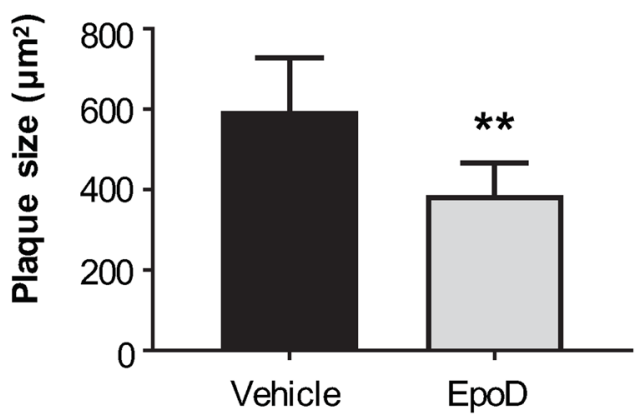

Figure 5. EpoD treatment reduced $A \beta$ pathology in APP/PS1 hippocampus. (a) Extracellular A $\beta$ accumulation was assessed by both A 342 immunohistochemistry $(\mathbf{a} 1, \mathbf{a} 2)$ and Thioflavin-S staining $(\mathbf{a} 3, \mathbf{a} 4)$ for fibrillary plaques in the CA1 subfield. Insets show higher magnifications of stained amyloid plaques. (b) Quantitative analyses demonstrated a significant reduction of $A \beta 42$ deposition in CA1 region $\left(t\right.$ test, $\left.{ }^{*} \mathrm{p}<0.05\right)$ of EpoD mice $(n=7)$ in comparison to vehicle group $(n=4)$. (c) Similarly, Thio-S loading was also found to be reduced in the hippocampus of EpoD treated mice $\left(t\right.$ test, $\left.{ }^{*} \mathrm{p}<0.05\right)$. (d) The numerical density (ND) of plaques did not change, but plaque size (e) was significantly smaller in EpoD group $\left(t\right.$ test, $\left.{ }^{* *} \mathrm{p}<0.01\right)$. slm stratum lacunosummoleculare, so stratum oriens, $s p$ stratum pyramidale, $s r$ stratum radiatum. Scale bars: (a1-a4) $200 \mu \mathrm{m}$; insets $20 \mu \mathrm{m}$. 
EpoD reduces both phospho-tau and $A \beta$ pathology. To determine the impact of EpoD treatment on the two major AD-pathological lesions we evaluated phospho-tau and $\mathrm{A} \beta$ accumulation in the hippocampus. This APP/PS1 model exhibits phospho-tau build-up within dystrophic neurites surrounding amyloid plaques $^{24,28,29}$. Thus, we first assessed the AT8/TAU46 ratio by western blot (Fig. 4a,b). As expected, there was a clear accumulation of AT8-positive tau in APP/PS1 ${ }^{\text {veh }}$ mice that was significantly reduced after EpoD treatment $(60.07 \pm 6.70 \%$ vs. $100 \pm 26.89 \%$, for EpoD or vehicle mice, respectively, $p<0.05)$. This decrease in phospho-tau levels was paralleled by a reduction on AT8-positive dystrophic neurites $(-27.17 \pm 19.1 \%, \mathrm{p}<0.05)$ around amyloid deposits in hippocampal CA1 region after EpoD treatment (Fig. 4c,d). Hence, these data indicate that EpoD treatment attenuated tau pathology.

Next, we analyzed the extracellular $\mathrm{A} \beta$ accumulation in the CA1 subfield by $\mathrm{A} \beta 42$ immunohistochemistry (Fig. 5a1,a2; hippocampal panoramic images are shown in Supplemental Fig. S3) as well as using the conformation-dependent dye Thioflavin-S for fibrillar amyloid species (Fig. 5a3,a4). With both approaches (Fig. 5b,c), extracellular $\mathrm{A} \beta$ burden was found to be slightly but significantly diminished after the EpoD treatment $(-19.5 \pm 17.02 \%$ for $A \beta 42$ and $-27.75 \pm 20.06 \%$ for Thio-S, $p<0.05)$. Stereological quantification of plaque numerical density (plaques $/ \mathrm{mm}^{2}$ ) in CA1 region (Fig. $5 \mathrm{~d}$ ) demonstrated that the density of amyloid deposits did not significantly change after treatment, however, their size (Fig. 5e) was significantly smaller $(-35.07 \pm 13.46 \%, \mathrm{p}<0.01)$ in APP/PS1 ${ }^{\mathrm{EpoD}}$ group compared to vehicle mice. Furthermore, this decrease was concomitant with a reduction in hippocampal total monomeric A $\beta$ levels in APP/PS1 ${ }^{\mathrm{EpoD}}$ compared to APP/ $\mathrm{PS}^{\text {Veh }}$ mice $(66.93 \pm 9.40 \%$ vs. $100 \pm 14.69 \%, \mathrm{p}<0.01)$ measured by western blot (Fig. $\left.6 \mathrm{a} 1, \mathrm{a} 2\right)$.

In order to further investigate this effect on $A \beta$ production, we next analyzed the intracellular $A \beta$ accumulation on synaptosomes isolated from Vehicle and EpoD-treated APP/PS1 mice (Fig. 6b1,b2). We have previously demonstrated ${ }^{29}$ that $\mathrm{A} \beta$ accumulates in the synaptosomal fraction and this accumulation could reflect the axonal pathology in this model. Thus, the microtubule/axonal stabilization could reduce the $A \beta$ content within the presynaptic terminals. As shown (Fig. 6b1,b2), EpoD-treated mice exhibited a striking reduction on synaptosomal $\mathrm{A} \beta$ content compared to vehicle group $(41.62 \pm 15.35 \%$ vs. $100 \pm 12.91 \%$, for EpoD or vehicle groups, respectively $\mathrm{p}<0.001)$. We next evaluated whether this decrease in the total or synaptosomal $\mathrm{A} \beta$ accumulation was also reflected by a reduction on the soluble oligomeric $A \beta$ forms. To test this possibility, the $S 1$ soluble fractions were isolated and the $A \beta$ oligomers were assayed using anti-oligomeric $O C$ and $A 11$ antibodies in dot blot experiments (Fig. 6c1,c2). As expected from previous works, S1 fractions isolated from APP/PS1 ${ }^{\text {veh }}$ mice accumulated both OC- and A11-positive $A \beta$ oligomers. These $A \beta$ oligomeric forms were highly reduced $(18.41 \pm 6.25 \%$ vs. $100.00 \pm 47.35 \%, p=0.0006 ; 14.18 \pm 4.22 \%$ vs. $100.30 \pm 23.62, p=0.0001$ for OC and A11 in EpoD or vehicle groups, respectively) in S1 fractions isolated from EpoD treated APP/PS1 mice.

Aiming to confirm that microtubule stabilization could indeed reduce the $\mathrm{A} \beta$ production, we have treated N2a-APP cell line with EpoD and the intracellular A $\beta$ content was tested by western blots. Even using this in vitro system and an acute treatment ( $12 \mathrm{~h}$ or $24 \mathrm{~h}$ of treatment, Supplemental Fig. S5 a1 and a2), EpoD produced a significant reduction on the intracellular A $\beta$ in N2a-APP cell line $(41.63 \pm 4.92 \%$ or $48.71 \pm 4.27 \%$ vs. $100.01 \pm 23.71 \%$ for $12 \mathrm{~h}$ and $24 \mathrm{~h} \mathrm{EpoD}$ vs. Control, respectively; $\mathrm{p}<0.05)$. It is also noteworthy that APP-CTFs were not significantly altered under these experimental conditions (Supplemental Fig. S5 d1 and d2). Thus, even under in vitro conditions, EpoD produced an appreciable effect on $A \beta$ production.

These data could be explained by a direct inhibitory effect of EpoD on either BACE- 1 or $\gamma$-secretase activities. Therefore, we tested by in vitro assays the possible inhibitory effect of EpoD on both APP-processing enzymatic activities. As shown (Fig. 6d), BACE-1 activity was not directly affected by EpoD treatment. The absence of BACE-1 inhibition is also consistent with the absence of modifications in the soluble APP $\beta$ or in C99-CTF fragments (Supplemental Fig. S5 c1, c2 and d1, d2, respectively). Similarly, we observed no changes on soluble APPa or C83-CTFs fragments, indicating the absence of $\alpha$-secretase inhibition (Supplemental Fig. S5 c1, c2 and d1, d2, respectively). Moreover, as shown in Fig. 6e1,e2, EpoD had not any appreciable direct effect on $\gamma$-secretase activity.

Together, these data revealed that microtubule stabilization by EpoD administration reduced in APP/PS1 mice the synaptic pathology, dystrophic neurites formation, SOM pathology and $A \beta$ accumulation, producing a preservation of hippocampal-dependent memory. EpoD also reduced the $A \beta$ production in an amyloidogenic cell model. This effect on A $\beta$ pathology was not mediated by altering BACE1 or $\gamma$-secretase activities.

\section{Discussion}

Many neurodegenerative pathologies, including Alzheimer's disease (AD), share microtubule (MT) abnormalities and axonal transport disruption ${ }^{15,18}$. Consequently, MT-targeting agents are attractive therapeutic candidates for these devastating brain disease ${ }^{20}$ and several clinical trials to evaluate microtubule stabilizers efficacy are currently ongoing ${ }^{21}$. MT-stabilizing drugs have been previously tested in Tau transgenic mouse models resulting in improved axonal transport and motor function ${ }^{41,42}$. However, the impact of MT-stabilizers on A $\beta$ pathology has not been assessed in vivo yet.

To the best of our knowledge, this is the first preclinical study using a brain-penetrant MT-stabilizer in an amyloidogenic transgenic model of Alzheimer's disease. We demonstrate that early administration of EpoD prevented cognitive decline and ameliorated AD-like pathology in the hippocampus of APP/PS1 mice. Besides the expected beneficial impact on tau pathology, EpoD treatment reduced the extracellular and presynaptic $A \beta$ accumulation. Moreover, EpoD administration decreases axonal/synaptic damage, dystrophic neurites formation and neuronal loss. Therefore, our data support that destabilization of cytoskeleton integrity is implicated in the progression of $\mathrm{A} \beta$ pathology and neuronal vulnerability.

Microtubules are critical for neuronal function and survival ${ }^{10,43}$. Post-translational modifications of tubulin are involved in the regulation of microtubule dynamics, constituting a useful code of functional information ${ }^{44,45}$. 
a1

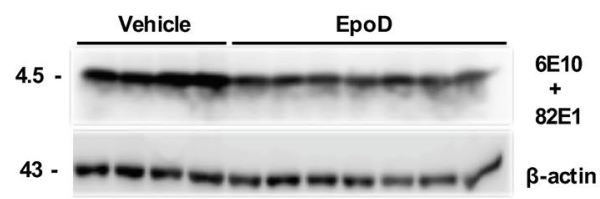

b1

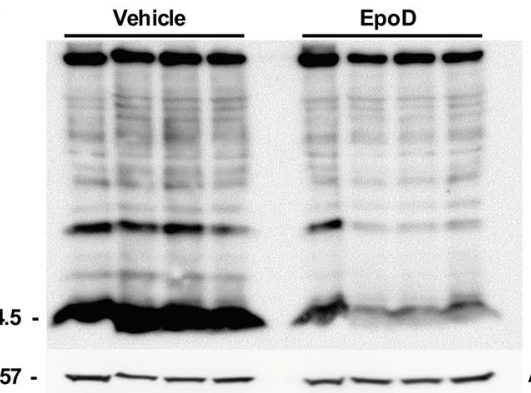

a2

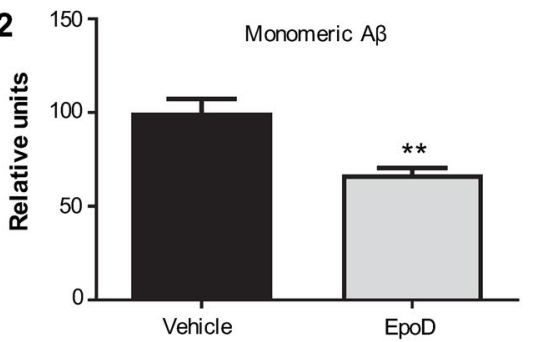

b2

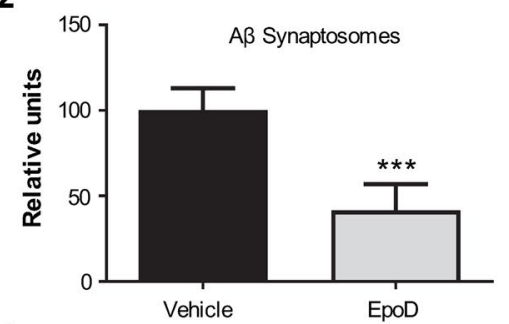

c1

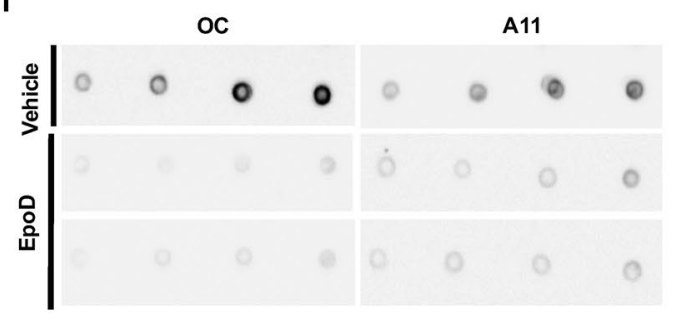

c2

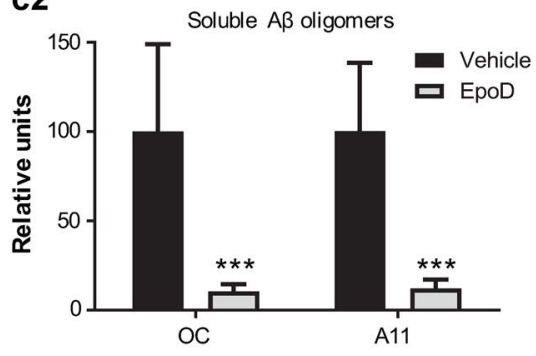

d

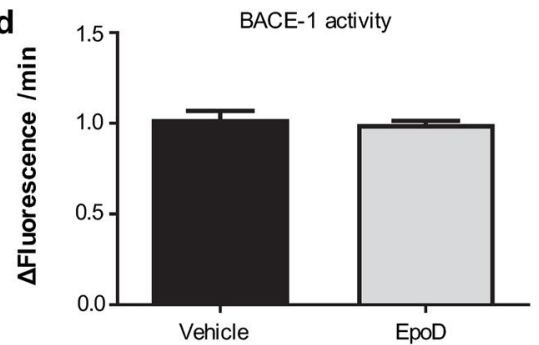

e1

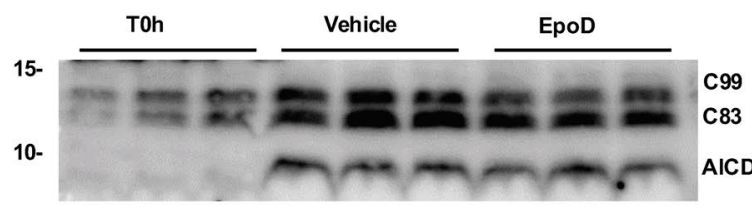

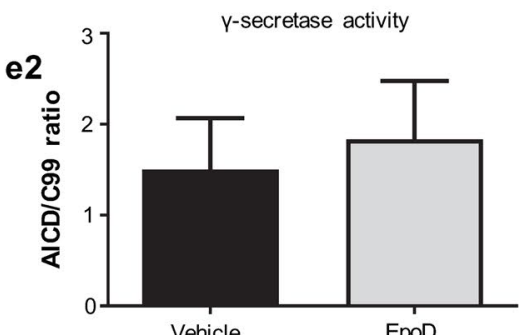

Vehicle

EpoD

Figure 6. Effect of EpoD treatment on the $\mathrm{A} \beta$ production. (a1,a2) Quantitative western blots using $6 \mathrm{E} 10$ plus $82 \mathrm{E} 1$ antibodies in total proteins isolated from hippocampus of vehicle $(n=4)$ and EpoD mice $(n=7)$. APP/PS1 ${ }^{\mathrm{EpoD}}$ animals showed a significant reduction of monomeric $A \beta$ levels as compared with the vehicle group $\left(t\right.$ test, $\left.{ }^{* *} \mathrm{p}<0.01\right)$. For quantification, levels were referred to vehicle group. (b1,b2) Importantly, western blotting of isolated hippocampal synaptosomes using the same antibodies demonstrated a stronger reduction in $\mathrm{A} \beta$ content in this neuronal fraction of EpoD mice $\left(t\right.$ test, ${ }^{* * *} \mathrm{p}<0.001$; $\mathrm{n}=4$ mice/group). (c1,c2) EpoD treatment also produced a strong reduction $\left({ }^{* *} \mathrm{p}<0.001\right)$ on the soluble oligomeric $\mathrm{A} \beta$ accumulation. A $\beta$ oligomers were assayed using OC or A11 dot blots. Equivalent S1 proteins from WT was used as negative controls. Protein loading was controlled using parallel dot-blots developed using ant-GAPDH (Supplemental Fig. S4). Direct effect of EpoD on BACE-1 (d) and $\gamma$-secretase $(\mathbf{e} 1, \mathbf{e} 2)$ activities. No significant differences were observed $(\mathrm{n}=6$ independent experiments). Uncropped blots are shown in Supplementary Fig. S9. 
For instance, the most stable pools, long-lived microtubules, are enriched in acetylated tubulins which protect them from mechanical aging ${ }^{37,38}$. A reduction of acetylated tubulin levels has been reported in AD brains ${ }^{46}$. In addition, it has been shown that $\mathrm{A} \beta$ increases MT instability ${ }^{47}$. In agreement with these observations, the expression of acetylated $\alpha$-tubulin was low in our transgenic APP/PS1 mice, indicating that unstable and depolymerized MTs may also contribute to AD pathology in this amyloidogenic model. Importantly, EpoD treatment resulted in an enrichment of acetylated tubulin and thus in the amount of stable MTs, as reported previously in models of tauopathy and Parkinson's disease $\mathrm{e}^{48-50}$. Then, this could translate into improved MT function and axonal transport in EpoD mice, and consequently, lead to reduced AD-pathology and clinical manifestations.

In line with this, the peripheral administration of EpoD, at the initial stages of the pathology, prevented the hippocampal-related cognitive deficits displayed by this amyloidogenic transgenic model. Similarly, EpoD treatment has proven to produce cognitive recovery in tauopathy models ${ }^{42,48,50}$. It is well established that synaptic damage, induced by soluble $\mathrm{A} \beta$ oligomers, is the best correlate of cognitive deficiencies in early stages of $\mathrm{AD}^{51,52}$, and our APP/PS1 model displays hippocampal synaptic alterations since early ages (this work; see also ${ }^{28}$ ). Therefore, EpoD treatment may promote cognitive function improvement in our model by preserving the synaptic integrity through microtubular stabilization, either directly enhancing the intracellular trafficking of protein cargos, or indirectly via a reduction of $\mathrm{A} \beta$ production or accumulation. EpoD seems to act both pre- and post-synaptically, increasing the levels of both synaptophysin and PSD95 proteins, respectively, and this effect could be probably due to the improvement of the microtubular-dependent traffic in both axonal and dendritic compartments. In this sense, it has been shown that EpoD reverses the A $\beta$-induced spine loss in organotypic cultures from APP hippocampus ${ }^{53}$. Also, an increase of the synaptic density was found in the CA3 subfield after EpoD treatment in a tauopathy model ${ }^{50}$. On the other hand, it has been demonstrated that during synapse elimination in the neuromuscular junction, the axonal branches dismantle their microtubules, and that the injection of Epothilone $\mathrm{B}$ is enough to delay this process ${ }^{54}$, highlighting the involvement of microtubular stabilization in synaptic maintenance. We have previously demonstrated that most of dystrophic neurites in APP/PS1 mice correspond to aberrant axons/presynaptic terminals ${ }^{27,28,31}$, which are characterized by cytoskeletal defects ${ }^{29}$ and accumulation of $\mathrm{A} \beta$ peptides $^{28,29,31}$. Interestingly, after EpoD treatment we detected a significant decrease in the dystrophic neurite number, the accumulation of ubiquitinated proteins, and the presynaptic $\mathrm{A} \beta$ content. Therefore, the restauration of microtubular integrity, together with the reduction of phospho-tau, probably ameliorate the axoplasmic flow and the synaptic preservation. Thus, it is highly likely that the synaptic recovery, linked to the reduction of axonal pathology, might explain the behavioral improvement after the EpoD treatment.

In addition, the close spatiotemporal relationship between all dystrophic neurites and amyloid plaques ${ }^{55}$ indicates that there is a direct connection between both pathological events. In fact, A $\beta$ is able to disrupt MTs ${ }^{47,56,57}$, thus promoting dystrophies formation, and dystrophies could be a source of $A \beta$ production $^{28,29,58}$, leading to a self-perpetuating cycle. Here, we found that EpoD treated mice show a significant reduction of both intracellular and extracellular A $\beta$ content in the hippocampus. Moreover, the treatment induced a reduction in plaque size. We have confirmed this effect of EpoD on $\mathrm{A} \beta$ accumulation using an independent Alzheimer's model. EpoD-treated N2a-APP cell cultures resulted in significant lower A $\beta$ levels. However, the $\beta$ - and $\gamma$-secretase activities were not altered by EpoD. Therefore, the decrease in A $\beta$ intracellular content, in the absence of C99 levels modification, might be explained by an EpoD mediated effect on intracellular vesicular transport, decreasing the probability of C99 and $\gamma$-secretase subcellular co-localization at synapses, and in consequence, decreasing the intracellular A $\beta$ production ${ }^{59}$. Alternatively, EpoD-mediated MT stabilization, by improving the axonal transport, may increase the $\mathrm{A} \beta$ degradation at the lysosomal compartment. Both circumstances agree with the drastic reduction on the synaptosomal $A \beta$ content, and the soluble $A \beta$ oligomeric forms, observed in this work after EpoD treatment.

A relevant finding of this study is the preservation of the highly vulnerable somatostatin-expressing interneurons in the EpoD treated animals. This neuronal population is extensively damaged in the APP/PS1 mouse, and the loss of somatostatin neurons has been closely associated to the $A \beta$ burden $^{30,31,33-35}$. In this regard, it is important to mention that, in this specific APP/PS1 model, only principal neurons are able to express the mutated human transgene ${ }^{28,34,35}$, and thus the SOM pathology is induced by the extracellular A $\beta$. Therefore, the effect of EpoD on the interneurons survival could be indirectly mediated by the A $\beta$ diminution, or interneuron preservation could also reflect an improvement in axonal transport, reduced intracellular protein accumulation and synaptic protection. Present results do not allow us to discriminate if the EpoD-protective effect is due to the reduction on extracellular A $\beta$ accumulation or the amelioration of MT instability. Probably both effects, reduction on plaques size together with diminution on the SOM-positive dystrophies, are implicated on the protection of this vulnerable population. To the best of our knowledge, this is the first report showing that EpoD prevents neuronal loss in an in vivo model of amyloidosis. EpoD-mediated SOM cell protection could explain, at least in part, the cognitive recovery in treated mice. GABAergic dysfunction has been postulated as major contributor for the cognitive impairment manifested by AD patients ${ }^{60}$. SOM neuron loss is a well-established pathogenic event in $\mathrm{AD}$ brains ${ }^{35,61-63}$. Considering that SOM participates in synaptic plasticity and memory process ${ }^{64-67}$, the loss of these interneurons may contribute to the cognitive deficits associated to $\mathrm{AD}$. In addition, the EpoD-induced SOM protection may also contribute to the reduced amyloid pathology observed in the treatment group mice. SOM regulates the levels of the $A \beta$-cleaving enzyme neprilysin ${ }^{68}$, interferes with the $A \beta$ fibrillization ${ }^{69}$ and regulates the $A \beta$-induced blood-brain barrier permeability ${ }^{70}$, indicating that the loss of this GABAergic subpopulation plays a pivotal role in $\mathrm{AD}$ pathogenesis.

In sum, our results indicated that either microtubule stabilization or the indirect effects induced by EpoD treatment promoted spatial memory, synaptic/neuritic recovery and neuronal protection. Furthermore, EpoD treatment reduced not only the tau pathology, but also, and more interestingly and novel, the presynaptic and extracellular accumulation of A $\beta$. Thus, MT-stabilizing compounds should be considered as therapeutic candidates to modify $\mathrm{AD}$ progression, targeting both $\mathrm{A} \beta$ and tau pathology. 


\section{Materials and methods}

Transgenic mice and Epothilone D treatment. Generation and characterization of APP ${ }^{751 S L} / \mathrm{PS}^{\mathrm{M} 146 \mathrm{~L}}$ (APP/PS1) has been previously reported ${ }^{24,28,31,32,34,35,71}$. These APP/PS1 mice were obtained by crossing heterozygotic Thy1-APP ${ }^{751 S L}$ (Swedish-K670N, M671L- and London-V717I-FAD mutations) mice with homozygous PS1 ${ }^{\mathrm{M} 146 \mathrm{~L}}$ mice (Charles River, France). Male and female 3-month-old APP/PS1 mice were randomly assigned to two groups to receive weekly intraperitoneal injections of $2 \mathrm{mg} / \mathrm{kg}$ Epothilone D (EpoD; Med. Chem. Express, $\mathrm{USA})\left(\mathrm{APP} / \mathrm{PS} 1^{\mathrm{EpoD}}, \mathrm{n}=8\right)$ or vehicle solution $\left(\mathrm{APP} / \mathrm{PS} 1^{\mathrm{Veh}}, \mathrm{n}=7\right)$ over a period of 3 months. Vehicle solution consisted of 1:1 (v/v) mixture DMSO/saline $(\mathrm{NaCl})$ to reduce DMSO toxicity. Animals were fed ad libitum with standard mice diet (2014 Teklad Global 14\% Protein Rodent Maintenance Diet, Harlan, Spain). Signs of abnormal behavior, distress, and body weight were weekly monitored. The EpoD treatment was well tolerated and no significant decrease in survival rate or weight was detected. All animal experiments were performed in accordance with the Spanish and the European Union regulations (RD53/2013 and 2010/63/UE) and approved by the Animal Research Committee from the University of Malaga (Spain). Experiments and procedures with animals were designed to minimize animal suffering and reduce the number of animals used.

Behavioral studies. During the last 2 weeks of the treatment, 6-month-old APP/PS1 ${ }^{\mathrm{EpoD}}(\mathrm{n}=8)$ and APP/ $\mathrm{PS}^{\mathrm{Veh}}(\mathrm{n}=7)$ mice were submitted to motor function and cognitive tests. Non-transgenic wild-type $(\mathrm{WT} ; \mathrm{n}=12)$ mice of the same genetic background and age were also included. Behavioral tests were performed during the light period of the light/dark cycle and the experimenter was blind to the genotypes and treatment of mice. Habituation was performed as described earlier ${ }^{30}$. All behavioral registrations were performed using the monitoring software Ethovision XT 7.0 (Noldus, Netherlands).

Open-field test (OFT) was used to examine motor function ${ }^{30,72}$ by means of spontaneous locomotor activity and vertical exploratory behavior or rearings. Mice were placed in a square-shape arena $(30 \times 30 \times 15 \mathrm{~cm})$ and allowed to explore for $5 \mathrm{~min}$. The locomotor activity was measured as the distance travelled and navigation speed with the software, while vertical exploratory behavior was quantified using an observational method.

Object recognition test. The object location task (OLT) and the novel object recognition task (NORT) were used to evaluate hippocampus-dependent spatial and non-spatial memory, respectively ${ }^{73,74}$. Both tests were performed 1 and $24 \mathrm{~h}$ after the habituation to the open-field. Both tasks consisted of an acquisition and a discrimination phase (test phase), of $10 \mathrm{~min}$ each one, with a delay of $3 \mathrm{~h}$ between them. During OLT, two identical objects were presented to the animals and in the test phase, one of the objects was displaced to a novel location. For NORT, mice are allowed to explore two identical sample objects. Then, one of the familiar objects was replaced by a novel one. The basal measure was the time spent by the mice exploring objects during the sample phases and the test trial. Additionally, two discrimination indexes were calculated for the test trial: a location recognition ratio (total time exploring displaced object/total time of exploration) and an object recognition ratio (total time exploring novel object/total time of exploration). The time was recorded only when the mice touched the object with their nose.

Y-maze specific context. This test was used to evaluate the long-term memory considering how familiar the animal finds a specific context ${ }^{75,76}$. The maze consists of three acrylic arms $(31 \times 18 \times 32 \mathrm{~cm})$ placed at $120^{\circ}$ respect to each other, with different internal visual clues. Animals performed two trials (training and test), with a $24 \mathrm{~h}$ inter-trial interval. During training, one arm was blocked off with a panel (novel arm). The mice were placed and allowed to freely explore the other two arms for $8 \mathrm{~min}$. In the test trial, all arms were accessible and mice explore freely for $5 \mathrm{~min}$. Time spent by the animal in each arm, distance $(\mathrm{cm})$, and speed $(\mathrm{cm} / \mathrm{s})$ were analyzed.

Morris water maze (MWM). This test evaluates spatial cognition and memory ${ }^{77,78}$ using a circular pool (1.4 m diameter, San Diego Instruments, Inc., California, USA), where animals have to locate a hidden platform beneath opaque water, guided by some spatial cues ${ }^{79,80}$. Mice were trained to find the platform (learning or acquisition phase) for 7 days (4 trials/day, maximum trial duration $90 \mathrm{~s}$, intertrial interval of $5 \mathrm{~min}$ ). On the day $7,1.5 \mathrm{~h}$ after the last trial, the platform was removed and animals were allowed to explore the maze for $60 \mathrm{~s}$ (retention phase). To discard visual or motivational deficiencies, on day 8 animals performed the visible platform test (4 trials, maximum trial duration $60 \mathrm{~s}$ ). Latency, distance travelled, speed, and time spent in the target quadrant (retention phase) were analyzed.

Tissue preparation. Three days after the last day of the treatment, mice were anesthetized with sodium pentobarbital $(60 \mathrm{mg} / \mathrm{kg})$ and transcardially perfused with $0.1 \mathrm{M}$ phosphate buffered saline (PBS). Then, brain was quickly removed; left hippocampus was dissected out and frozen while right hemisphere was fixed by immersion with $4 \%$ paraformaldehyde, $75 \mathrm{mM}$ lysine, $10 \mathrm{mM}$ sodium metaperiodate in $0.1 \mathrm{M}$ phosphate buffer (PB), pH 7.4 for 5 days at $4{ }^{\circ} \mathrm{C}$. Fixed hemispheres were cryoprotected in $30 \%$ sucrose, coronally sectioned at $40 \mu \mathrm{m}$ thickness in a freezing microtome and serially collected in cold PBS and $0.02 \%$ sodium azide.

Antibodies. The following primary antibodies and dilutions were used: anti-A $\beta 42$ rabbit polyclonal (1:5,000, Abcam); anti-A $\beta$ (clone $6 \mathrm{E} 10)$ mouse monoclonal (1:5,000, Signet); anti-human A $\beta$, N-terminus (clone 82E1) mouse monoclonal (1:6,000, IBL); anti-acetylated $\alpha$-tubulin (clone 6-11B-1) mouse monoclonal (1:50,000, Sigma); anti-human amyloid precursor protein (hAPP) rabbit polyclonal (1:20,000, Sigma); anti-ATP synthase $\beta$ (clone 10/ATP) mouse monoclonal (1:10,000, Brand BD Transduction Laboratories); anti- $\beta$-Actin 
(clone AC 74) mouse monoclonal (1:10,000, Sigma-Aldrich); anti-phospho-PHF-tau pSer202/Thr205 (clone AT8) mouse monoclonal (IHC 1:1,000, Cell Signaling); anti-PSD95 rabbit monoclonal (1:2,000, Cell signaling); anti-Somatostatin (SOM) goat polyclonal (1:1,000, Santa Cruz Biotechnology); anti-synaptophysin rabbit polyclonal (1:1,000, Abcam); anti-total tau (TAU46) mouse monoclonal (1:1,000, Cell Signaling) and anti-ubiquitin rabbit polyclonal (1:5,000, Dako).

Total protein extraction and Western blots. Total protein was extracted as described previously ${ }^{29,34,71,81}$. Protein pellets from dissected hippocampi, obtained using the Tripure TM Isolation Reagent, were resuspended in $4 \% \mathrm{SDS}$ and $8 \mathrm{M}$ urea in $40 \mathrm{mM}$ Tris- $\mathrm{HCl}, \mathrm{pH} 7.4$ and rotated overnight at room temperature. Western blots (WB) were performed as described previously ${ }^{82}$. Briefly, 7-15 $\mu \mathrm{g}$ of protein from the different samples were loaded on $10 \%$ and $12 \%$ SDS-Tris-Glycine-PAGE and transferred to nitrocellulose (Hybond-C Extra, Amersham, Sweden). To analyze A $\beta$, protein samples were loaded onto $16 \%$ SDS-Tris-Tricine-PAGE and transferred to PVDF (Inmobilon-P, Millipore). After blocking, using 5\% non-fat milk, the membranes were incubated overnight, at $4{ }^{\circ} \mathrm{C}$, with the appropriate antibody. Membranes were then incubated with the corresponding horseradish-peroxidase (HRP)-conjugated secondary antibody (Cell Signaling) at a dilution of 1:10,000. Each blot was developed using the ECL-plus detection method (Amersham, Sweden). For quantification, images were obtained using Image-Quant Las 4000 mini gold (GE Healthcare Bio-Sciences) and analyzed using PCBAS program. In each experiment, the intensity of bands from WT mice and/or experimental condition were averaged and considered as $100 \%$ relative units. For normalization purposes, proteins were first estimated by Lowry and protein loading corrected by beta-actin. Data were always normalized by the specific signal observed in 6-month-old WT group.

Synaptosomes isolation. The synaptosomal fractions were obtained as described previously ${ }^{28}$. Briefly, the tissue was homogenized using a Dounce homogenizer in $0.32 \mathrm{M}$ Sucrose, $10 \mathrm{mM}$ Tris- $\mathrm{HCl}$ (pH 7.4) buffer (buffer A) containing complete protease and phosphatase inhibitor cocktails (Sigma). After homogenization, the crude synaptosomal fraction (synaptosomes plus mitochondria) was isolated by two sequential centrifugations $\left(1,500 \times g, 10 \mathrm{~min}\right.$ followed by $12,500 \times \mathrm{g}, 20 \mathrm{~min}$; at $\left.4{ }^{\circ} \mathrm{C}\right)$. The crude synaptosomes were resuspended in $13 \%$ (final concentration) Ficoll 400 (in buffer A) and layered on the bottom of a discontinuous gradient, composed by buffer A and 7\% Ficoll (in buffer A). The gradients were centrifuged at $100,000 \times g\left(45 \mathrm{~min}\right.$ at $4{ }^{\circ} \mathrm{C}$ ) and the synaptosomes were isolated at the 7.5-13\% interface. After washing (twice with buffer A), the protein content of the synaptosomal fractions was quantified by Lowry.

BACE-1 and $y$-secretase activity determination. BACE-1 activity was determined using a commercial kit (R\&D Systems, Germany) following the manufacturer instructions. Briefly, fresh hippocampal membranes were solubilized in the buffer supplied by the manufacturer, centrifuged at $10,000 \times g\left(15 \mathrm{~min}\right.$ at $\left.4{ }^{\circ} \mathrm{C}\right)$ and the supernatant (100-200 $\mu \mathrm{g}$ of protein) was used for BACE-1 assay. The $\gamma$-secretase activity was determined as described previously ${ }^{29}$. Briefly, membranes, isolated from APP/PS1 cortex, were resuspended (at $3 \mathrm{mg}$ of protein per $\mathrm{ml}$ ) in $150 \mathrm{mM}$ Citrate Buffer, $\mathrm{pH}$ 6.4, containing protease inhibitors (Roche). Aliquots ( $150 \mu \mathrm{g}$ of proteins) were used for each assay. Samples were then incubated, at $37^{\circ} \mathrm{C}$ with orbital shaking, for $2 \mathrm{~h}$ in absence or in presence of Epothilone D $(100 \mathrm{nM})$. After incubation, membranes were sonicated (at $80 \mathrm{~W}$ for $30 \mathrm{~s}$ ) and centrifuged at $30,000 \times g\left(30 \mathrm{~min}, 4^{\circ} \mathrm{C}\right)$. Supernatants were used to determine AICD production by western blot using anti-APP C-terminal antibody. As negative control, membranes were keep on ice $\left(4^{\circ} \mathrm{C}\right)$ and treated as above.

APPswe-expressing N2a cultures. APPswe-stably transfected Neuroblastoma cells were generously donated by Dr. Gopal Thinakaran (University of Chicago).

N2aAPPswe cells were cultured as described ${ }^{29}$. For EpoD treatment, a $0.2 \mu \mathrm{m}$ filtered stock solution of Epothilone D was diluted in the same media at a final concentration of $100 \mathrm{nM}$. This media was kept for 12 or $24 \mathrm{~h}$ before collecting the cells and isolating RNA and protein as described above.

Immunohistochemistry. The immunolabeling procedures were done as described earlier ${ }^{28-34}$. Serial sections from all animals were processed in parallel for immunostaining using the same batches of solutions to minimize variability during immunostaining processing. Free-floating sections were first treated with $3 \% \mathrm{H}_{2} \mathrm{O}_{2} / 10 \%$ methanol in PBS, pH 7.4 for 20 min to inhibit endogenous peroxidases, and with avidin-biotin Blocking Kit (Vector Labs, Burlingame, CA, USA) for $30 \mathrm{~min}$ to block endogenous avidin, biotin and biotin binding proteins. After primary antibody incubation ( $24 \mathrm{~h}$ at room temperature), sections were incubated with the corresponding biotinylated secondary antibody (1:500 dilution, Vector Laboratories) followed by streptavidin conjugated HRP (1:2000, Sigma-Aldrich). The peroxidase reaction was visualized with $0.02 \% 3$-3-diaminobenzidine tetrahydrochloride (DAB, Sigma-Aldrich), $0.03 \%$ nickel ammonium sulphate and $0.01 \%$ hydrogen peroxide in PBS. Sections immunolabeled for APP, AT8, ubiquitin or SOM were incubated in $0.2 \%$ Congo-red solution. For immunofluorescent labeling, sections were incubated with the primary antibody followed by Alexa 568 secondary antibody (1:1,000, Invitrogen). Specificity of the immune reactions was controlled by omitting the primary antisera. For Thioflavin-S staining, free-floating sections were incubated for 5 min with $0.015 \%$ Thio-S (Sigma) in 50\% ethanol, and then rinsed in 50\% ethanol and PBS. Quantitative comparisons were carried out on sections processed at the same time with same batches of solutions.

Image analysis. Plaque loading (percentage of total CA1 area A $\beta 42$-immunopositive or stained with Thioflavin-S), dystrophic neurite loading (percentage of total CA1 area covered by APP-positive or Ubiquitin-positive 
dystrophic neurites) and Synaptophysin-positive area (ratio of immunopositive area of the molecular sublayer/ molecular layer area) were quantified. All image quantification was performed as previously reported ${ }^{30,31,33,35}$. Immunostained sections (4 sections/animal) from $\operatorname{APP} / P S 1^{\mathrm{EpoD}}(\mathrm{n}=7)$ and $\mathrm{APP} / \mathrm{PS}^{\mathrm{Veh}}(\mathrm{n}=4)$ mice were visualized under a Nikon Eclipse 50i microscope coupled with a Nikon DS-5M high-resolution digital camera. ACT$2 \mathrm{U}$ program (Auto Camera Tame to You, Imaging software, Nikon Corporation 2004) was used to take digital images of hippocampal regions using a $10 \times$ objective. Thioflavin-S staining was examined under an Olympus BX-61 epifluorescent microscope using FITC filter and $4 \times$ objective. Images were acquired with an Olympus DP71 high-resolution digital camera using the Cell-A program (Olympus). The camera settings were adjusted at the start of the experiments and maintained for uniformity. Digital images were analyzed using Visilog 6.3 analysis program (Noesis, France). Staining densities were identified by level threshold, which were maintained throughout the experiment for consistency. Grey-scale images were converted to binary images. The sums were averaged and a single plaque burden was computed for each mouse.

The CA1 area or dentate gyrus molecular layers in each image was manually outlined and in the case for dystrophic neurite loading the positive somata were removed by manual editing.

Stereological analysis. Neuron and dystrophic neurites counting. SOM-positive cells and SOM- or AT8positive dystrophic neurites were stereologically quantified in the CA1 subfield of APP/PS1 ${ }^{\mathrm{EpoD}}(\mathrm{n}=7)$ and APP/ $P S 1^{\text {Veh }}(n=4)$ groups according to the optical fractionator method as described previously ${ }^{30,31,33-35}$. Wild-type mice $(n=4)$ were used as control group for SOM-cell counting. Briefly, the quantitative analyses were performed using an Olympus BX61 microscope interfaced with a computer and an Olympus DP71 digital camera, and the NewCAST (Computer Assisted Stereological Toolbox) software package (Olympus, Denmark). Cell/dystrophic quantification was done through the rostrocaudal extent of the hippocampus (between $-0.94 \mathrm{~mm}$ anterior and $3.64 \mathrm{~mm}$ posterior to Bregman coordinates), in every seventh section (with a distance of $280 \mu \mathrm{m}$ ), and an average of 7 sections were analyzed for each animal. CA1 boundaries were defined using a $4 \times$ objective and the number of neurons was counted using a $100 \times / 1.35$ objective. We used a counting frame of $1874.2 \mu \mathrm{m}^{2}$ with step lengths of $68.45 \times 68.45 \mu \mathrm{m}$. The numerical density (ND; SOM cells or dystrophies $/ \mathrm{mm}^{3}$ ) of immunopositive events was estimated using the following formula: $\mathrm{ND}=\mathrm{N} /\left(\mathrm{A} \times 10 \_\mathrm{m} / \mathrm{SV}\right)$, where $\mathrm{N}$ is the number of dissectorcounted somatic/dystrophic profiles, A, the area and SV is the volumetric shrinkage factor of the sample (the SV was determined in the same way as described by ${ }^{34}$. The precision of the individual estimations is expressed by the coefficient of error (CE). Total CE (CE group value) was calculated using the CEs in each individual animal. An investigator who was blinded to the experimental conditions performed neuronal/dystrophic profile counts.

Plaque size. Plaque morphometric analysis was performed as previously reported ${ }^{30} \mathrm{using}$ the nucleator method with isotropic probes by the NewCAST software package from Olympus stereological system. CA1 subfield was analyzed in sections from APP/PS1 $1^{\mathrm{Veh}}(\mathrm{n}=4)$ and $\mathrm{APP} / \mathrm{PS} 1^{\mathrm{EpoD}}(\mathrm{n}=7)$ mice immunostained with anti-A $\beta 42$ using a counting frame of $6,022.8 \mu \mathrm{m}^{2}$. For individual plaque measurement, a $40 \times$ objective was used. Each analysis was done by a single examiner blinded to sample identities.

Statistical analysis. Statistical analyzes were performed using the GraphPad software 7.0. Data are presented as mean \pm standard deviation (SD), except for the cognitive data expressed as mean \pm standard error of the mean (SEM). The comparison between two groups was done by two-tailed Student's $t$ test. To compare several groups, we used one-way ANOVA and one-way or two-way ANOVA with repeated measures (RM) followed by Tukey post-hoc test. The significance was set at $95 \%$ of confidence.

\section{Data availability}

All data generated in this study are included in this article and its online supplementary information.

Received: 29 November 2019; Accepted: 18 August 2020

Published online: 08 September 2020

\section{References}

1. Götz, J., Halliday, G. \& Nisbet, R. M. Molecular pathogenesis of the tauopathies. Annu. Rev. Pathol. Mech. Dis. 14, 239-261 (2018).

2. Henstridge, C. M., Hyman, B. T. \& Spires-Jones, T. L. Beyond the neuron-cellular interactions early in Alzheimer disease pathogenesis. Nat. Rev. Neurosci. 20, 94-108 (2019).

3. Long, J. M. \& Holtzman, D. M. Alzheimer disease: an update on pathobiology and treatment strategies. Cell 179, 312-339 (2019).

4. Webers, A., Heneka, M. T. \& Gleeson, P. A. The role of innate immune responses and neuroinflammation in amyloid accumulation and progression of Alzheimer's disease. Immunol. Cell Biol. https://doi.org/10.1111/imcb.12301 (2019).

5. Shi, Y. \& Holtzman, D. M. Interplay between innate immunity and Alzheimer disease: APOE and TREM2 in the spotlight. Nat. Rev. Immunol. 18, 759-772 (2018).

6. Navarro, V. et al. Microglia in Alzheimer's disease: activated, dysfunctional or degenerative. Front. Aging Neurosci. https://doi. org/10.3389/fnagi.2018.00140 (2018).

7. Salter, M. W. \& Stevens, B. Microglia emerge as central players in brain disease. Nat. Med. 23, 1018-1027 (2017).

8. Bodakuntla, S., Jijumon, A. S., Villablanca, C., Gonzalez-Billault, C. \& Janke, C. Microtubule-associated proteins: structuring the cytoskeleton. Trends Cell Biol. 29, 804-819 (2019).

9. Goodson, H. V. \& Jonasson, E. M. Microtubules and microtubule-associated proteins. Cold Spring Harbor Perspect. Biol. https:// doi.org/10.1101/cshperspect.a022608 (2018).

10. Guedes-Dias, P. \& Holzbaur, E. L. F. Axonal transport: driving synaptic function. Science https://doi.org/10.1126/science.aaw 99 97 (2019).

11. Park, J. H. \& Roll-Mecak, A. The tubulin code in neuronal polarity. Curr. Opin. Neurobiol. 51, 95-102 (2018).

12. Cuchillo-Ibanez, I. et al. Phosphorylation of tau regulates its axonal transport by controlling its binding to kinesin. FASEB J. 22, 3186-3195 (2008). 
13. D’Amelio, M. \& Rossini, P. M. Brain excitability and connectivity of neuronal assemblies in Alzheimer's disease: from animal models to human findings. Prog. Neurobiol. 99, 42-60 (2012).

14. Nixon, R. A. \& Yang, D. S. Autophagy failure in Alzheimer's disease-locating the primary defect. Neurobiol. Dis. 43, 38-45 (2011).

15. Matamoros, A. J. \& Baas, P. W. Microtubules in health and degenerative disease of the nervous system. Brain Res. Bull. 126, 217-225 (2016).

16. Adalbert, R. \& Coleman, M. P. Axon pathology in age-related neurodegenerative disorders. Neuropathol. Appl. Neurobiol. 39, 90-108 (2013).

17. Bakota, L. \& Brandt, R. Tau biology and tau-directed therapies for Alzheimer's disease. Drugs 76, 301-313 (2016).

18. Brunden, K. R., Lee, V. M., Smith, A. B., Trojanowski, J. Q. \& Ballatore, C. Altered microtubule dynamics in neurodegenerative disease: therapeutic potential of microtubule-stabilizing drugs. Neurobiol. Dis. 105, 328-335 (2017).

19. Madav, Y., Wairkar, S. \& Prabhakar, B. Recent therapeutic strategies targeting beta amyloid and tauopathies in Alzheimer's disease. Brain Res. Bull. 146, 171-184 (2019).

20. Varidaki, A., Hong, Y. \& Coffey, E. T. Repositioning microtubule stabilizing drugs for brain disorders. Front. Cell. Neurosci. 12, 226 (2018).

21. Congdon, E. E. \& Sigurdsson, E. M. Tau-targeting therapies for Alzheimer disease. Nat. Rev. Neurol. 14, 399-415 (2018).

22. Brunden, K. R., Lee, V. M., Smith, A. B., Trojanowski, J. Q. \& Ballatore, C. Neurobiology of disease altered microtubule dynamics in neurodegenerative disease: therapeutic potential of microtubule-stabilizing drugs. Neurobiol. Dis. https://doi.org/10.1016/j. nbd.2016.12.021 (2016).

23. Zhao, Y., Mu, X. \& Du, G. Pharmacology and therapeutics microtubule-stabilizing agents: new drug discovery and cancer therapy. Pharmacol. Ther. 162, 134-143 (2016).

24. Blanchard, V. et al. Time sequence of maturation of dystrophic neurites associated with A $\beta$ deposits in APP/PS1 transgenic mice. Exp. Neurol. 184, 247-263 (2003).

25. Caballero, C. et al. Inter-individual variability in the expression of the mutated form of hPS1M146L determined the production of A $\beta$ peptides in the PS1xAPP transgenic mice. J. Neurosci. Res. 85, 787-797 (2007).

26. Gavilán, M. P. et al. Molecular and cellular characterization of the age-related neuroinflammatory processes occurring in normal rat hippocampus: potential relation with the loss of somatostatin GABAergic neurons. J. Neurochem. 103, 984-996 (2007).

27. Gomez-Arboledas, A. et al. Phagocytic clearance of presynaptic dystrophies by reactive astrocytes in Alzheimer's disease. Glia 66, 637-653 (2018).

28. Sanchez-Varo, R. et al. Abnormal accumulation of autophagic vesicles correlates with axonal and synaptic pathology in young Alzheimer's mice hippocampus. Acta Neuropathol. 123, 53-70 (2012).

29. Torres, M. et al. Defective lysosomal proteolysis and axonal transport are early pathogenic events that worsen with age leading to increased APP metabolism and synaptic Abeta in transgenic APP/PS1 hippocampus. Mol. Neurodegener. 7, 59 (2012).

30. Trujillo-Estrada, L. et al. In vivo modification of Abeta plaque toxicity as a novel neuroprotective lithium-mediated therapy for Alzheimer's disease pathology. Acta Neuropathol. Commun. 1, 73 (2013).

31. Trujillo-Estrada, L. et al. Early neuronal loss and axonal/presynaptic damage is associated with accelerated amyloid- $\beta$ accumulation in AßPP/PS1 Alzheimer's disease mice subiculum. J. Alzheimer's Dis. 42, 521-541 (2014).

32. Baglietto-Vargas, D. et al. Calretinin interneurons are early targets of extracellular amyloid- $\beta$ pathology in PS1/A $\beta$ PP Alzheimer mice hippocampus. J. Alzheimer's Dis. 21, 119-132 (2010).

33. Moreno-Gonzalez, I. et al. Extracellular amyloid- $\beta$ and cytotoxic glial activation induce significant entorhinal neuron loss in young PS1M146L/APP751SL mice. J. Alzheimer's Dis. 18, 755-776 (2009).

34. Ramos, B. et al. Early neuropathology of somatostatin/NPY GABAergic cells in the hippocampus of a PS1 $\times$ APP transgenic model of Alzheimer's disease. Neurobiol. Aging 27, 1658-1672 (2006).

35. Sanchez-Mejias, E. et al. Distinct disease-sensitive GABAergic neurons in the perirhinal cortex of Alzheimer's mice and patients. Brain Pathol. https://doi.org/10.1111/bpa.12785 (2019).

36. Lou, K. et al. Brain-penetrant, orally bioavailable microtubule-stabilizing small molecules are potential candidate therapeutics for Alzheimer's disease and related tauopathies. J. Med. Chem. 57, 6116-6127 (2014).

37. Schulze, E., Asai, D. J., Bulinski, J. C. \& Kirschner, M. Posttranslational modification and microtubule stability. J. Cell Biol. 105, 2167-2177 (1987).

38. Portran, D., Schaedel, L., Xu, Z., Théry, M. \& Nachury, M. V. Tubulin acetylation protects long-lived microtubules against mechanical ageing. Nat. Cell Biol. 19, 391-398 (2017).

39. Deller, T., Martinez, A., Nitsch, R. \& Frotscher, M. A novel entorhinal projection to the rat dentate gyrus: direct innervation of proximal dendrites and cell bodies of granule cells and GABAergic neurons. J. Neurosci. 16, 3322-3333 (1996).

40. Bakst, I., Avendano, C., Morrison, J. H. \& Amaral, D. G. An experimental analysis of the origins of somatostatin-like immunoreactivity in the dentate gyrus of the rat. J. Neurosci. 6, 1452-1462 (1986).

41. Zhang, B. et al. Microtubule-binding drugs offset tau sequestration by stabilizing microtubules and reversing fast axonal transport deficits in a tauopathy model. Proc. Natl. Acad. Sci. 102, 227-231 (2005).

42. Barten, D. M. et al. Hyperdynamic microtubules, cognitive deficits, and pathology are improved in tau transgenic mice with low doses of the microtubule-stabilizing agent BMS-241027. J. Neurosci. 32, 7137-7145 (2012).

43. Kelliher, M. T., Saunders, H. A. \& Wildonger, J. Microtubule control of functional architecture in neurons. Curr. Opin. Neurobiol. 57, 39-45 (2019)

44. Song, Y. \& Brady, S. T. Post-translational modifications of tubulin: pathways to functional diversity of microtubules. Trends Cell Biol. 25, 125-136 (2015).

45. Janke, C. \& Kneussel, M. Tubulin post-translational modifications: encoding functions on the neuronal microtubule cytoskeleton. Trends Neurosci. 33, 362-372 (2010).

46. Hempen, B. \& Brion, J.-P. Reduction of acetylated $\alpha$-tubulin immunoreactivity in neurofibrillary tangle-bearing neurons in Alzheimer's disease. J. Neuropathol. Exp. Neurol. 55, 964-972 (1996).

47. Tsushima, H. et al. HDAC6 and RhoA are novel players in Abeta-driven disruption of neuronal polarity. Nat. Commun. https:// doi.org/10.1038/ncomms8781 (2015).

48. Brunden, K. R. et al. Epothilone D improves microtubule density, axonal integrity, and cognition in a transgenic mouse model of tauopathy. J. Neurosci. 30, 13861-13866 (2010).

49. Cartelli, D. et al. Microtubule alterations occur early in experimental parkinsonism and the microtubule stabilizer Epothilone D is neuroprotective. Sci. Rep. 3, 1837 (2013).

50. Zhang, B. et al. The microtubule-stabilizing agent, epothilone D, reduces axonal dysfunction, neurotoxicity, cognitive deficits, and alzheimer-like pathology in an interventional study with aged tau transgenic mice. J. Neurosci. 32, 3601-3611 (2012).

51. Walsh, D. M. \& Selkoe, D. J. Deciphering the molecular basis of memory failure in Alzheimer's disease. Neuron 44, 181-193 (2004).

52. Arendt, T. Synaptic degeneration in Alzheimer's disease. Acta Neuropathol. 118, 167-179 (2009).

53. Penazzi, L. et al. A $\beta$-mediated spine changes in the hippocampus are microtubule-dependent and can be reversed by a subnanomolar concentration of the microtubule-stabilizing agent epothilone D. Neuropharmacology 105, 84-95 (2016).

54. Brill, M. S. et al. Branch-specific microtubule destabilization mediates axon branch loss during neuromuscular synapse elimination. Neuron 92, 845-856 (2016). 
55. Meyer-Luehmann, M. et al. Rapid appearance and local toxicity of amyloid- $\beta$ plaques in a mouse model of Alzheimer's disease. Nature 451, 720-724 (2008).

56. Zempel, H., Thies, E., Mandelkow, E. \& Mandelkow, E.-M. Abeta oligomers cause localized Ca(2+) elevation, missorting of endogenous Tau into dendrites, Tau phosphorylation, and destruction of microtubules and spines. J. Neurosci. 30, 11938-11950 (2010).

57. Zempel, H. et al. Amyloid- $\beta$ oligomers induce synaptic damage via Tau-dependent microtubule severing by TTLL6 and spastin. EMBO J. 32, 2920-2937 (2013).

58. Sadleir, K. R. et al. Presynaptic dystrophic neurites surrounding amyloid plaques are sites of microtubule disruption, BACE1 elevation, and increased A $\beta$ generation in Alzheimer's disease. Acta Neuropathol. 132, 235-256 (2016).

59. Sannerud, R. et al. Restricted location of PSEN2/ $\gamma$-secretase determines substrate specificity and generates an intracellular A $\beta$ pool. Cell 166, 193-208 (2016).

60. Palop, J. J. \& Mucke, L. Network abnormalities and interneuron dysfunction in Alzheimer disease. Nat. Rev. Neurosci. 17, 777-792 (2016).

61. Burgos-Ramos, E. et al. Somatostatin and Alzheimer's disease. Mol. Cell. Endocrinol. 286, 104-111 (2008).

62. Epelbaum, J. et al. Somatostatin, Alzheimer's disease and cognition: an old story coming of age?. Prog. Neurobiol. 89, 153-161 (2009).

63. Kumar, U. Expression of somatostatin receptor subtypes (SSTR1-5) in Alzheimer's disease brain: an immunohistochemical analysis. Neuroscience 134, 525-538 (2005).

64. Liguz-Lecznar, M., Urban-Ciecko, J. \& Kossut, M. Somatostatin and somatostatin-containing neurons in shaping neuronal activity and plasticity. Front. Neural Circuits 10, 1-15 (2016).

65. Yavorska, I. \& Wehr, M. Somatostatin-expressing inhibitory interneurons in cortical circuits. Front. Neural Circuits 10, 1-18 (2016).

66. Abbas, A. I. et al. Somatostatin interneurons facilitate hippocampal-prefrontal synchrony and prefrontal spatial encoding. Neuron 100, 926-939.e3 (2018).

67. Kim, D. et al. Distinct roles of parvalbumin- and somatostatin-expressing interneurons in working memory. Neuron 92, 902-915 (2016).

68. Saito, T. et al. Somatostatin regulates brain amyloid beta peptide Abeta42 through modulation of proteolytic degradation. Nat. Med. 11, 434-439 (2005)

69. Solarski, M., Wang, H., Wille, H. \& Schmitt-Ulms, G. Somatostatin in Alzheimer's disease: a new role for an old player. Prion 12, $1-8(2018)$.

70. Paik, S., Somvanshi, R. K. \& Kumar, U. Somatostatin maintains permeability and integrity of blood-brain barrier in $\beta$-amyloid induced toxicity. Mol. Neurobiol. 56, 292-306 (2019).

71. Jimenez, S. et al. Inflammatory response in the hippocampus of PS1M146L/APP 751SL mouse model of Alzheimer's disease: agedependent switch in the microglial phenotype from alternative to classic. J. Neurosci. 28, 11650-11661 (2008).

72. Filali, M. et al. Cognitive and non-cognitive behaviors in the triple transgenic mouse model of Alzheimer's disease expressing mutated APP, PS1, and Mapt (3xTg-AD). Behav. Brain Res. 234, 334-342 (2012).

73. Ennaceur, A. \& Delacour, J. A new one-trial test for neurobiological studies of memory in rats. 1: behavioral data. Behav. Brain Res. 31, 47-59 (1988).

74. Davis, K. E., Easton, A., Eacott, M. J. \& Gigg, J. Episodic-like memory for what-where-which occasion is selectively impaired in the 3xTgAD mouse model of Alzheimer's disease. J. Alzheimer's Dis. 33, 681-698 (2013)

75. Hughes, R. N. The value of spontaneous alternation behavior (SAB) as a test of retention in pharmacological investigations of memory. Neurosci. Biobehav. Rev. 28, 497-505 (2004).

76. Karl, T., Bhatia, S., Cheng, D., Kim, W. S. \& Garner, B. Cognitive phenotyping of amyloid precursor protein transgenic J20 mice. Behav. Brain Res. 228, 392-397 (2012).

77. Morris, R. Developments of a water-maze procedure for studying spatial learning in the rat. J. Neurosci. Methods 11, 47-60 (1984).

78. Navarrete, F. et al. Métodos de evaluación de trastornos cognitivos en modelos animales. Rev. Neurol. 47, 137-145 (2008).

79. Malleret, G., Hen, R., Guillou, J. L., Segu, L. \& Buhot, M. C. 5-HT1B receptor knock-out mice exhibit increased exploratory activity and enhanced spatial memory performance in the Morris water maze. J. Neurosci. 19, 6157-6168 (1999).

80. Hendershott, T. R., Cronin, M. E., Langella, S., McGuinness, P. S. \& Basu, A. C. Effects of environmental enrichment on anxietylike behavior, sociability, sensory gating, and spatial learning in male and female C57BL/6J mice. Behav. Brain Res. 314, 215-225 (2016).

81. Jimenez, S. et al. Age-dependent accumulation of soluble amyloid $\beta(A \beta)$ oligomers reverses the neuroprotective effect of soluble amyloid precursor protein- $\alpha$ (sAPP $\alpha$ ) by modulating phosphatidylinositol 3-kinase (PI3K)/Akt-GSK- $3 \beta$ pathway in Alzheimer mouse model. J. Biol. Chem. 286, 18414-18425 (2011).

82. Araujo, F. et al. Molecular and pharmacological characterization of native cortical $\gamma$ - aminobutyric acids receptors containing both $\alpha 1$ and $\alpha 3$ subunits. J. Biol. Chem. 271, 27902-27911 (1996).

\section{Acknowledgements}

This study was supported by Instituto de Salud Carlos III (ISCiii) of Spain, co-financed by FEDER funds from European Union, through grants PI15/00796 and PI18/01557 (both to AG), PI15/00957 and PI18/01556 (both to JV), and CIBERNED (CB06/05/1116 to AG and CB06/05/0094 to JV); by Malaga University grant PPIT.UMA. B1.2017/26 (to RSV). JJFV and CMC were supported by FPU PhD fellowships (Spanish Ministry of Science, Innovation and Universities). RSV held a postdoctoral contract from Malaga University. IMG is recipient of a senior postdoctoral contract from Ramon y Cajal Program (Spain Government). We thank Sanofi (France) for the APP/PS1 model used in this work. We thank Dr. G. Thinakaran for the generous gift of transfected N2a-APPsew cells. In addition, we would also like to thank Mercedes Aneiros Ferrer for her excellent technical support.

\section{Author contributions}

A.G., J.V., and R.S.V. conceived the study, analyzed and interpreted data, wrote the manuscript and secured the funding. J.J.F.V. and V.D.C. performed the behavioral analysis. J.J.F.V. carried out the histology and immunohistochemical studies, designed the figures and participated in writing. C.M.C., V.N. and S.J. performed western blots and contributed to data analysis. E.S.M., C.N.D., and A.G.A. assisted with image and stereological analysis. M.V., J.C.D. and I.M.G. critically revised the manuscript for intellectual content. All authors read and approved the final manuscript before submission.

\section{Competing interests}

The authors declare no competing interests. 


\section{Additional information}

Supplementary information is available for this paper at https://doi.org/10.1038/s41598-020-71767-4.

Correspondence and requests for materials should be addressed to J.V. or A.G.

Reprints and permissions information is available at www.nature.com/reprints.

Publisher's note Springer Nature remains neutral with regard to jurisdictional claims in published maps and institutional affiliations.

Open Access This article is licensed under a Creative Commons Attribution 4.0 International License, which permits use, sharing, adaptation, distribution and reproduction in any medium or format, as long as you give appropriate credit to the original author(s) and the source, provide a link to the Creative Commons licence, and indicate if changes were made. The images or other third party material in this article are included in the article's Creative Commons licence, unless indicated otherwise in a credit line to the material. If material is not included in the article's Creative Commons licence and your intended use is not permitted by statutory regulation or exceeds the permitted use, you will need to obtain permission directly from the copyright holder. To view a copy of this licence, visit http://creativecommons.org/licenses/by/4.0/.

(c) The Author(s) 2020 